(Aus dem Physiologischen Institut der deutschen Universität in Prag.)

\title{
Messende Untersuchungen über den Geltungsgrad spektraler Farbengleichungen.
}

\section{Zugleich ein Beitrag zur Experimentalkritik der Dreikomponenten- lehre des Farbensinnes.}

\author{
Von
}

H. Goldmann,

Assistent am Physiologischen Institut.

Mit 9 Textabbildungen.

(Eingegangen am 14. Januar 1922.)

\section{Das Problem des Geltungsgrades optischer Gleichungen.}

Die Methodik unserer Untersuchungen besteht darin, daß bei gegebener Ursache und gegebenem Effekt dieser als charakteristisches Bestimmungsstück des Substrates, an dem er auftritt, gilt. Man kann dabei ganz absehen von Differentialvorgängen an Teilen des Substrates, die erst in ihrer Reihe zu dem untersuchten Effekt führen, man muß nur dann den Effekt als Gesamtwirkung am Gesamtsubstrat, nicht nur an dem letztbetroffenen Teil, bezeichnen. So ist die bei Einwirkung von Licht auf das Auge resultierende Empfindung nicht Wirkung des Lichtes auf die Netzhaut, noch auf die Hirnrinde, sondern Wirkung auf das ,Sehorgan“. Alles, was sich bei phänomenologisch-analytischer Untersuchung der Empfindung ${ }^{1}$ ) nicht in Beziehung zu. Eigentümlichkeiten des Reizes setzen läßt, ist spezifisch für das Substrat; seine Charakteristik läßt im Substrat begründete, in unserem Falle also physiologische Gesetzmäßigkeiten klar hervortreten. Es ist dies jene Betrachtungsweise, welche $A$. Tschermak ${ }^{2}$ ) als ,exakten Subjektivismus" bezeichnet hat. Überall dort, wo wir lieber von "Auslösung" als von Ursache reden, tritt besonders klar hervor, wie wir aus der Diskrepanz von Ursache und Effekt Substratmerkmale bestimmen. Wenn man also den Reiz und den Reizeffekt einzeln definiert hat, so lassen

1) Vgl. spez. E. Mach, Analyse der Empfindungen. 8. Aufl. Jena 1919.

$\left.{ }^{2}\right)$ A. Tschermak, Der exakte Subjektivismus in der neueren Sinnesphysiologie. Berlin, Springer 1921 (sep. aus Pflügers Arch. f. d. ges. Physiol. 188, 1. 1921). 
sich aus all dem, was Charakteristisches an diesem und nicht an jenem auftritt, Schlüsse auf physiologische Eigenheiten des ,Sehorgans" ziehen. Würden hingegen Erscheinungen z. B. nach der Strahlungsintensität allein sich ändern, so wären diese Änderungen offenbar völlig durch den Reiz bestimmt, wie durch Transformation aus ihm ableitbar. Die Sinnesphysiologie verlangt also einerseits Definiertheit des Reizes, andererseits Phänomenologie und Analyse der Empfindung, und charakterisiert damit das sich ändernde Substrat, das Sinnesorgan. Aber da die Empfindung offenbar Ausdruck eines physiologischen Geschehens ist, gibt sie allein schon Aufschluß über physiologische Vorgänge; nur läßt sich, wie $v$. Kries betont, von vornherein nicht aussagen, ob dabei auftretende Besonderheiten gerade von dem Wege im Sehorgan und nicht von anderen Faktoren abhängig sind [Gedächtnis, Sprachgebrauch usw. $\left.{ }^{1}\right)$ ]. Wie immer dem sei, wenn wir Reiz und Reizeffekt in Funktionsbeziehung setzen, werden sich auch darüber Aussagen machen lassen.

Für die Gesichtsempfindungen ergibt sich dabei alsbald die Schwierigkeit, daß sie kein eigenes Maßsystem besitzen, da sie ja Qualitätsverschiedenheiten darstellen ${ }^{2}$ ). Man kann sie also von vornherein nicht in Reihen ordnen, in denen ein Glied zahlenmäßig aus einem anderen abgeleitet werden kann; man kann nur Ähnlichkeitsreihen oder Reihen von Relationen zu gewissen einfachsten oder Grundempfindungen aufstellen. In diesen Reihen vollzieht sich das Ordnen nach denselben allgemeinen Gesetzen wie bei einer mathematischen Reihe. Auch hier sind die Dimensionen des Systems bestimmt, wenn man seine unabhängig Variablen kennt. Als Variable unseres Systems betrachten wir mit E. Hering ${ }^{3}$ ) die als Farbenton, Sättigung und Nuance bezeichneten Attribute einer farbigen Gesichtsempfindung. Jeder Lichtreiz ist definiert durch die Wellenlängen des Strahlungsinhaltes und durch die jeder Wellenlänge zugeordnete Intensität. Es ist dies mit allen möglichen Kombinationen eine unendliche Mannigfaltigkeit. Das erste, was sich bezüglich der Reizeffekte oder Empfindungen feststellen läßt, ist die Einschränkung der unendlichen Mannigfaltigkeit auf eine dreifache im obigen Sinne. Wenn man nun - wie dies früher geschehen daraufhin den Reiz definiert sein läßt durch Wellenlänge, Mischung mit weißem Licht und Intensität, und dann feststellt, daß eine ganz ähnliche Gliederung in der Empfindung vorliegt, so ist das eigentlich ein Hineintragen des Empfindungsinhaltes in den Reiz, eine antizipative

I) J. v. Kries, Handbuch der Physiologie, herausgeg. von W. A. Nagel, III (1), S. 138. Braunschweig, Vieweg. 1905.

2) Vgl. spez. A. Tschermak, a. a. O., spez. S. 5, 6.

3) Vgl. die bezügliche Auseinandersetzung mit C. Stumpf bei A. Tschermak, a. a. O., S. 6, Anm 1 . 
Einschränkung, und die nachherige Übereinstimmung darf nicht wundernehmen ${ }^{1}$ ).

Die allgemeine Darstellung einer Empfindung in obigem System von 3 Mannigfaltigkeiten ist demnach $M\left(x_{a}, y_{a}, z_{a}\right)$. Es bleibt noch die Frage nach dem Verhältnis der subjektiven Helligkeit ejner Gesichtsempfindung zu dem dreidimensionalen System zu beantworten. Hat man einen „Körper“" 2) in diesem System so liegen, daß durch ihn die Nuanceachse hindurchgeht, so kann man ihn in Flächen schneiden, die gleicher Nuance sind (und zwar Pyramidenmäntel). Andere Flächen, in unserem Falle Mantelflächen eines Prismas, haben gleiche Sättigung. Endlich gibt es Flächen zweier komplementärer Farben. Man kann nun in diesem System auch Flächen gleicher Helligkeit legen. Diese lassen sich als Flächen eines anderen Koordinatensystems betrachten, das man durch Umformung aus dem ersten gewinnen kann, von dem aber für uns nur die eine Koordinate bedeutsam ist. Sie läßt sich also

1) Wäre das weiße Licht etwas Fixes, so hätte dieses Vorgehen eine gewisse Berechtigung; nun ist dies aber nicht der Fall, wie Hering immer und immer wieder betont (speziell in: Newtons Gesetz der Lichtermischung, Lotos, N. F. 7, 48. 1887; Individuelle Verschiedenheiten des Farbensinnes, ebenda 6, 28ff. 1885). Setzt man aber ein Lichtgemisch ein für allemal als "Weiß" fest, so muß man jedes andere auf dieses beziehen, was darauf hinausläuft, daß man ein Lichtersystem nur allgemein nach Wellenlängen und zugehörigen Intensitäten charakterisieren kann.

2) Während oben der allgemein geläufige Farblzörper, in deın jeder Punkt eine Farbe vorstellt, benutzt wurde (vgl. speziell W. Ostwald, ,Mathematische Farbenlehre", Leipzig 1918. Unesma), verwendet für gewisse Zwecke A. Tschermak mit Vorteil zur Veranschaulichung und schematischen Einordnung der Mannigfaltigkeit der Farbentöne, Sättigungsstufen und Nuancen häufig folgendes Schema. In einem dreidimensionalen Koordinatensystem mit vertikaler Weiß-Schwarzachse, transversaler Rot-Grünachse und anteroposteriorer Gelb-Blauachse wird eine vertikale Strecke - veranschaulicht durch einen Stab - von fixer Länge so verschieblich gedacht, daß sie nur im Grenzfalle bloß der oberen oder bloß der unteren Raumhälfte zugehört, während ihr sonst somit stetsein Projektionsabschnitt sowohl auf die obere weiße Halbachse wie auf die untere schwarze Halbachse zukommt. Das Verhältnis dieser beiden Streckenanteile (W : S) bezeichnet die Nuance. Das Verhältnis der Summe der Projektionen auf die rote oder grüne und die gelbe oder blaue Halbachse zur Streckenlänge, also $(R+G e)$ oder $(R+B l)$ : $(W+S)$, gibt eine k]are Charakteristik für die Sättigungsstufe [eigentlich $(R+G e):(R+G e+W+S)]$; das Verhältnis der Projektionen auf zwei farbige Halbachsen ( $R: G e$ oder $R: B l$ ) entspricht dem Farbenton. Nuancengleich sind demgemä $\beta$ alle einer horizontalen Schicht angehörenden Lagen, welche die Stabstrecke ohne Vertikalverschiebung einnehmen kann. Sättigungsgleich sind alle Streckenlagen in der Mantelfläche eines um die Weiß- oder Schwarzachse gelegten vierkantigen Prismas (durch dessen Kanten die beiden Farbachsen gehen). Tongleich sind alle Streckenlagen in einer durch die Weiß-Schwarzachse gelegten Ebene. Die Helligkeit ist eine von Farbenton, Sättigung, Nuance abhängige, gemeinsame Funktion - etwa vergleichbar der durch verschiedenartige, gleichzeitig ablaufende Prozesse gemeinsam bestimmten absoluten Reaktion oder Wasserstoffionenkonzentration (A. Tschermak). 
aus den 3 Koordinaten des ersten Systems ableiten: $h=\varphi(F, S, N)$; das sagt aus, daß bei beliebiger Wabl der 3 unabhängig Variablen das System bestimmt ist. Ordnet man in der obigen Gleichung $h$ einen Wert zu, dann lassen sich nur noch 2 Variable frei wählen. Man kann so verschiedene Empfindungen auf eine der 3 unabhängig Variablen oder $h$ untersuchen und kommt so zum Begriffe des Geltungsgrades einer Gleichung.

Der Geltungsgrad farbloser optischer Gleichungen ist bei Übereinstimmung beider Hälften in der subjektiven Helligkeit, welche prinzipiell von der physikalischen, objektiven Lichtstärke oder Intensität $\mathrm{zu}$ unterscheiden ist, ein vollständiger - gleichgültig aus welchen Komponenten die verglichenen Gemische physikalisch zusammengesetzt sind. Solche Gleichungen erweisen sich zwar nicht von der objektiven Lichtstärke (wenigstens innerhalb der verwendeten Grenzen), wohl aber vom Adaptationszustande des beobachtenden Auges abhängig, indem bei fortschreitender Dunkeladaptation eine farblose Mischung aus Rot- und Grünlicht mehr an Helligkeit gewinnt als eine farblose Mischung aus Gelb- und Blaulicht. Aus diesem Verhalten ist eine charalkteristische Verschiedenheit der spektralen Weiss-Reizwertkurve für das Hellauge und für das Dunkelauge zu erschließen ${ }^{1}$ ).

Für den Geltungsgrad farbiger Gleichungen kommen neben der subjektiven Helligkeit die elementaren Empfindungsqualitäten Farbenton, Sättigung und Nuance in Betracht, wobei die letzteren beiden rein subjektiv, d. h. als durch die Quantität und die Qualität des der Farbe subjektiv beigemengten Grau charakterisierte Empfindungsattribute, gefaßt werden: Auf dem Gebiete farbiger Gleichungen ist auch ein auf Übereinstimmung in der Helligkeit beschränkter Geltungsgrad möglich, wenn auch die Ergebnisse solcher heterochromatischer Photometrie, sowohl was die durch die Einstellungsfehler charakterisierte Sicherheit anbelangt, als noch mehr, was die subjektive Bestimmtheit der Gleichsetzung betrifft, recht unvollkommen zu nennen sind.

Hier beschäftigt uns speziell das Problem des auf den Farbenton beschränkten, also unvollständigen Geltungsgrades farbiger Gleichungen. In praxi werden allerdings bei der Herstellung solcher Tongleichungen weitgehende gleichzeitige Differenzen an Helligkeit, Sättigung, Nuance vermieden, also eine weitgehende Beschränkung in der Mannigfaltigkeit

1) An Literatur bezüglich der adaptativen Abhängigkeit farbloser Gleichungen seien speziell folgende Arbeiten von A. T'schermak zitiert: Über die Bedeutung der Lichtstärke und des Zustandes des Sehorgans für farblose optische Gleichungen. Pflügers Arch. f. d. ges. Physiol. \%0, 297. 1897; Beobachtungen über die relative Farbenblindheit im indirekten Sehen. Ebenda 82. 559. 1900. Vgl. auch ,Die HellDunkeladaptation des Auges und die Funktion der Stäbchen und Zapfen", Ergebn. d. Physiol. 1 (2), 725 ff. 1902; Wie die Tiere sehen, verglichen mit dem Menschen. Schriften des Vereins für naturwissenschaftliche Kenntnisse. Verlag Braumüller, Wien 1914. 
der nicht dem Farbentone gleich, ,gebundenen " Empfindungsqualitäten miteingeführt.

Der Farbenton des Empfindungseffektes, den ein spektrales Licht erzeugt, ist bekanntlich - auch bei Konstantsetzen des Zustandes des beobachtenden Auges - im allgemeinen nicht eine einfache Funktion von dessen Wellenlänge, sondern ist auch von dessen Lichtstärke abhängig. Nur 3 ausgezeichnete Lichter im Spektrum, welche dem jeweiligen Urgelb, Urgrün, Urblau im Sinne Herings entsprechen, zeigen bei wachsender Intensität keine Änderung des Farbentones, sondern nur eine solche der Sättigung, Nuance und Helligkeit. Alle anderen, "mischfarbig" erscheinenden Lichter werden bei wachsender Lichtstärke relativ mehr gelblich als rötlich oder grünlich, bzw. relativ mehr bläulich als grünlich oder rötlich - unter gleichzeitiger Abnahme der Sättigung (jenseits eines gewissen Optimums), Hellerwerden der Nuance, Zunahme der Gesamthelligkeit. Allerdings ist diese Abstufung in praxi technisch ziemlich beschränkt, wenn auch an einer fortschreitenden Vergilbung (unter Abblassen) der Lichter von etwa 700 bis ca. $570 \mu \mu$ und von ca. 565 bis etwa 530 (oder 510) einerseits, an einer fortschreitenden Verbläuung (unter Abblassen) der Lichter von etwa 510 (oder 490 ) bis etwa 475 und von $465-400$ andererseits prinzipiell nicht zu zweifeln ist ${ }^{1}$ ).

Dementsprechend lassen sich Gleichungen mit einem auf Farbenton beschränkten Geltungsgrad sog. Tongleichungen in gewissem Umfange bereits zwischen zwei auf jeder Gleichungshälfte einzeln gebotenen homogenen Lichtern herstellen. Ja, man bezeichnet traditionell, wenn auch tatsächlich nicht so ganz zutreffend, die äußerste Rotstrecke von 700 bis etwa $640 \mu \mu$ schon auf der gewöhnlich gegebenen Intensitätsstufe geradezu als tongleich bzw. zu Gleichungen von vollkommenem Geltungsgrade verwendbar. Jedenfalls lassen sich innerhalb des Rotgelb mehr langwellige bzw. mehr rötliche Einzellichter durch bloße Steigerung der Lichtstärke auf Tongleichheit mit mehr kurzwelligen bzw. mehr gelblichen Lichtern bringen, wobei allerdings die Helligkeit (zugleich auch die Sättigung und Nuance) zunehmend verschieden ausfällt. Ganz Analoges gilt, wenn auch mit technischen Beschränkungen, für die gelbgrüne, die grünblaue und die violette Strecke des Spektrums. Grundsätzlich kann man jedoch wohl die These vertreten: ein mischfarbiges spektrales Licht gestattet die Herstellung einer Tongleichung

1) Der umgekehrte Fall des Röter- und Grünerwerdens bei Intensitätsminderung hat als Bezold-Briucke sches Phänomen (W. v. Bezold, Über das Gesetz der Farbenmischung und die physiologischen Grundfarben. Pogg. Ann. 150, 71 u. 221. 1873; E. v. Briicke, Über einige Empfindungen im Gebiete des Sehnerven. Sitzungsber. d. Wien. Akad, d. Wiss. Mathem.-naturw. Kl., Abb. III, ry, 39. 1878) bei der Suche nach den sog. Grundempfindungen der Young-Helmholtzschen Theorie eine gewisse Rolle gespielt. 
mit allen anderen Lichtern, welche auf derselben mischfarbigen Strecke, bzw. im gleichen mischfarbigen Quadranten, gegen das Urblau bzw. das Urgelb gelegen sind. Ist also ein homogenes Licht gegeben und läßt man seine Intensität wachsen, so wächst die Helligkeit; ob aber in $\varphi$ $(f, s, n)$ nur 1 Variable oder 2 oder alle 3 sich geändert haben, läßt sich daran nicht voraussagen. Der Augenschein lehrt, daß alle 3 sich ändern. Alle diese Änderungen liegen auf einer Kurve, sind eine einfache Mannigfaltigkeit, gerade so wie der Reiz, nur beiderseits begrenzt. Dabei ist also (Konstanz des beobachtenden Auges vorausgesetzt) eine völlig zwangläufige Verknüpfung der einzelnen Tonstufen mit ganz bestimmten Stufen der Sättigung, Nuance und Helligkeit gegeben; die Variation der über den Farbenton hinausliegenden Empfindungsqualitäten erweist sich als völlig ,gebunden".

Werden 2 Lichter verschiedener Wellenlänge und variabler Intensität auf derselben Gleichungshälfte geboten, so lassen sich alle Kombinationen auf einer Ebene darstellen. Es gelingt jetzt, an Farbentönen alle zu „mischen“, welche zwischen jenen der beiden Wellenlängen bei gegebenen Intensitätsstufen gelegen sind, - wenn die beiden Lichter in unterkomplementärem Abstand voneinander gelegen sind, beispielsweise tonreines, $d . h$. weder rötlich noch grünlichgelbes Licht (,,urgelbes" im Sinne $E$. Herings) und nicht tonreines blaues, sondern grünlich-blaues Licht gegeben ist. Die durch Intensitätsänderungen einer einzelnen Komponente hervorgerufene Änderungen des Farbentons lassen sich in diesem Falle durch Änderungen im Verhältnis der beiden Komponenten ausgleichen. Bei gerade komplementärem oder gegenfarbigem Abstand resultieren Abstufungen der Sättigung bis zu Farblosigkeit. Bei überkomplementärem Abstand erhält man bekanntlich die Folge der Farbentöne des kleineren Sektors des Farbenkreises, beispielsweise durch Mischung von gelbem und rötlich-blauem Licht die Violett-Purpur-Rotreihe bis zum Gelb. Beschränken wir uns auf die Mischung von 2 unterkomplementären oder untergegenfarbigen Lichtern, so läßt sich hier - im Gegensatze zu dem früher betrachteten einfachsten Falle, von Intensitätsänderung eines einzelnen Lichtes, in welchem sich Farbenton, Sättigung und Nuance absolut gekoppelt verhielten - bei Festhalten des Farbentones bereits eine große Mannigfaltigkeit von Sättigungs- und Nuancenstufen erzeugen ${ }^{1}$ ). Allerdings ist dieselbe auf der einen Seite durch ein nicht überschreitbares Sättigungsoptimum beschränkt; auch sind ohne kontrasterregende Mitwirkung eines zweiten Lichtreizes neben dem binären Lichtergemisch - nur die an Helligkeit über dem sog. Eigen-

1) Wie weitgehend bereits mit 2 Farben eine Annäherung an die Wirklich. keit möglich ist, beweist das von Urban und Smith ausgearbeitete „Kinemacolor". Verfahren, mit Hilfe von Orange- und Blaugrünfiltern farbenkinematographische Aufnahmen und Projektionen auszuführen (Naturwissenschaften 9, 790. 1921). 
grau gelegenen Nuancen möglich. Sättigung und Nuance sind nicht einfach unabhängig voneinander variabel, sondern stehen hier in einer komplexen Koppelung, so daß - infolge der mit der Lichtstärke anfangs wachsenden, weiterhin jenseits eines Optimums wieder abnehmenden Sättigung - prinzipiell 2 Nuancen, eine relativ dunkle und eine relativ helle, für jede Sättigungsstufe möglich ist. Die Helligkeit ist hier, wenn der Farbenton konstant gehalten wird: $\varphi(s, n)$; dabei ist etwa $s=\varphi^{\prime}\left(n^{2}\right)$ in unserem System. D. h. also: Ist die Helligkeit bekannt, so sind die Werte $s$ und $n$ mitgegeben, das System ist schon durch Farbenton und Helligkeit oder durch Farbenton und Nuance eindeutig bestimmt; es ist zweidimensional wie der Reiz. Alle erhaltbaren Kombinationen liegen somit auf einer abgegrenzten Fläche, die allerdings auch gekrümmt sein kann.

Werden uns endlich 3 Lichter von verschiedener Wellenlönge und variabler Intensität auf derselben Gleichungshälfte geboten, so besagt bekanntlich das Newton sche Mischungsgesetz, daß bezüglich des Farbentones der Gleichung nur dann keinerlei Bindung mehr besteht - also alle überhaupt möglichen Farbentöne erreichbar sind, wenn die 3 Lichter passend gewählt sind, d. h. tatsächlich: wenn in den von den 3 Lichtern einzeln hervorgebrachten Empfindungen bereits alle 4 möglichen Tonelemente der Farbenempfindung (Rot, Gelb, Grün, Blau) nachweisbar enthalten sind. Es darf daher nicht tonreines, d. h. weder gelbliches noch bläuliches Rot (Urrot im Sinne $E$. Herings) und tonreines Grün nebst Blau oder Grünblau oder Violett gewählt werden. Mindestens $\mathrm{muß}$ das rote oder das grüne Licht zugleich gelblich sein, um aus beiden durch Ändern des Intensitätsverhältnisses alle gelben Töne mischen zu können ${ }^{1}$ ). Wählt man beide Tichter gelblich, so gewinnt man zwar kein Plus an möglichen Farbentönen, wohl aber eine große Mannigfaltigkeit an möglichen Sättigungsstufen, welche auch leidlich gesättigte (nicht bloß recht unsatte!) Stufen einschließt. - Im Gegensatze zur Erschöpfung aller möglichen Farbentöne bei passender Wahl von 3 Lichtern ergibt sich auch noch in diesem Falle eine weitgehende Beschränkung oder Bindung bezüglich der Produzierbarkeit von Sättigungsstufen und Nuancen.

Mit einer Vierzahl von Lichtern wird die eben erwähnte Bindung in weiterem Umfange gelöst, was besonders durch die Produzierbarkeit höherer Sättigungsstufen der früher nur nebenbei vertreten gewesenen Farbe auffällt. Während bei Gegebensein von (gelblich-)

1) Es sei erinnert an $A$. Tschermaks Vergleich der Mischungserzeugung von Gelb mit der sog. Goldmacherei und an den Satz: „Eine solche 3-Lichter-Mischung bedeutet in Wahrheit eine 4-Farben-Mischung." Der exakte Subjektivismus in der neueren S nnesphysiologie. Sep. Berlin 1921 aus Pflügers Arch. f. d. ges. Physiol. 188 (1), spez. S. 4. 1921. 
rotem, (gelblich-)grünem und blauem Licht nur recht unsattes Gelb ,erzeugt" werden konnte, ist erst bei Verwendung von 4 passenden Lichtern auch ,gutes" Gelb zu erzielen - wie jeder kritische Beurteiler von 3-Farben-Photogrammen zugeben wird. Allerdings bedeutet auch die Vierzahl von nicht durchwegs urfarbigen Lichtern durchaus noch nicht die Möglichkeit von Erzeugung aller nur möglichen „Farben“; die Beschränkung bezüglich Sättigung und Nuance ist erheblich gemildert, aber nicht aufgehoben.

Doch für uns steht hier voran das Problem des Geltungsgrades farbiger binär-homogener Gleichungen, d. h. solcher, welche zwischen einer Mischung von 2 unterkomplementären Spektrallichtern und dazwischenliegenden beliebigen homogenen Strahlungen hergestellt werden können.

An früheren Beobachtungsdaten seien folgende in Erinnerung gerufen. Bereits 1852 bemerkte Helmholtz ${ }^{1}$ ), welcher als erster Spektrallichtergleichungen ingrößerem Umfange herstellte: "Rot und Gelb gibt ein Orange, dessen Ansehen dem einfachen Orange vollständig gleich erscheint, und ebenso können die aus Blau und Violett zusammengesetzten Arten des Indigoblau wohl kaum vom einfachen Indigoblau unterschieden werden. Dagegen gibt schon Gelbgrün und Blaugrïn ein Grün, dessen Farbenton dem des prismatischen mittleren Grün zwar entspricht, welches aber entschieden weißlicher und matter ist, so daß das einfache Grün nur aus solchen Farben gemischt werden kann, die sich fast gar nicht im Ansehen von ihm unterscheiden. Gelb und Blau erscheinen in dieser Beziehung weniger empfindlich als Grün. Ersteres setzt sich noch ziemlich gut aus Orange und Gelbgrün zusammen, wird aber sehr fahl aus Rot und Grün, letzteres läßt sich gut ans Blaugrün und Indigo zusammensetzen, wird aber sehr matt aus Grün und Violett." Maxwell ${ }^{2}$ ), der als erster genaue Untersuchungen über das Lichtermischungsgesetz anstellte und es richtig fand, sagte hingegen: „The orange and yellow of the spectrum are chromatically equivalent to mixtures of red and green. They are neither richer nor paler than the corresponding mixtures and the only difference is that the mixture may be resolved by a prism whereas the colour in the spectrum cannot be resolved. " - Analogerweise gibt $J . J$. Muiller ${ }^{3}$ ) an, bis zur Linie b $(517 \mu u)$ mit allen langwelligen Homogenlichtern vollständige Mischungsgleichungen erhalten zu haben, ebenso von Linie $F(485 \mu \mu)$ bis zum violetten Ende.

Im Jahre 1881 beschrieb Lord Rayleigh ${ }^{4}$ ) das verschiedene Verhalten von farbentüchtigen Individuen, welches wir heute als Ausdruck von 2 differenten Typen betrachten, beim Einstellen einer Gelbgleichung aus Gelblichrot + Grün $=$ Gelb. Donders ${ }^{5}$ ) untersuchte daraufhin an seinem Doppelspektroskop eine ganze Reihe von Personen, wobei er als erster die weiterhin als "Rayleigh-Gleichung" bezeichnete Gleichung ( $m$ Li [670,5] $+n T h[535]=o N a[589]$ ) verwendete, während Rayleigh nur beiläufig eine solche Gleichung benutzt hatte. Er fand, daß "Li-Rot und Th-Grün eine Mischung von Gelb liefern, deren Farbe dem

1) H. Helmholtz, Über die Theorie zusammengesetzter Farben. Habilitationsschrift. Königsberg 1852. bern

2) C. Maxwell, On the Theory of compound Colours and the Relations of the Colours of the Spectrum. Phil. Trans. 150, 57-84.

$\left.{ }^{3}\right)$ J. J. Mïller, Zur Theorie der Farben. Arch. f. Ophthalmol, 15, 208. 1869.

4) W. Rayleigh, Experiments on colour. Nature 25, $64 \mathrm{ff}$. 1881. Mitgeteilt in der British Assoc. 2. Sept. 1881.

5) F. C. Donders, Farbengleichungen. Du Bois Arch. 1884, S. $518 \mathrm{ff}$

Pelügers Archiv f. d. ges. Physiol. Bd. 194. 
$N \alpha$-Gelb vollkommen gleich ist und bei mittlerer Intensität an Sättigung wenig nachsteht".

E. Hering ${ }^{\prime}$ ) brachte dazu noch sehr interessante Beobachtungen bei. Er sagt: "Sehr eindringlich waren die Verschiedenheiten zwischen $B$. (relativ gelbsichtig), $S$. (relativ blausichtig gegen Hering) und mir, wenn ich für $B$. Gleichungen zwischen Grüngelb und einem Gemisch von Orange und gelblichem Grün machte. Für $B$. war eine solche Gleichung auch in betreff der Sättigung möglich; jch selbst sehe das Mischgrün in B.s. Gleichung weniger gesättigt und im Vergleich zu dem homogenen Grüngelb als reines Grün, $S$. aber sah das Mischgelb sogar bläulich-grün (durch Kontrast) und sehr auffallend weniger gesättigt. Eine Gleichung zwischen dem relativ satten Grüngelb und der weißlichen Farbe, welche für ihn aus der Mischang der beiden eben genannten Lichtarten entsteht, vermochte er überhaupt nicht mit konstantem Ergebnis einzustellen. - Aus Blaugrün oder Grünblau und Violett eine brauchbare Gleichung mit reinem homogenen Blau herzustellen, ist nicht gelungen, weil das Mischblau stets viel weniger gesättigt war als das homogene Blau. Sobald aber die Sättigungsdifferenz zu groß wird, nimmt auch die Zuverlässigkeit der Ergebnisse schnell ab. Das viel gesättigtere homogene Blau vernichtet in dem an und für sich viel weniger gesättigten Mischblau noch einen weiteren Teil der blauen Empfindung duroh den Kontrast, und so bleibt ein Blaugrau oder Graublau übrig, welches durch ganz geringfügige Nebenumstände sehr leicht ins Grünliche oder Rötliche umschlägt. Ist vollends das homogene Blau nicht ganz reines Blau, so wird die Verwicklung durch den Kontrast noch größer." "

Von $J$. 3. Kries ${ }^{2}$ ) und seinen Schülern, so speziell von $W . A$. Nagel ${ }^{3}$ ) wurde bekanntlich die sog. Rayleigh-Gleichung trotz ihrer Unvollkommenbeit vielfach zur Diagnose der sog. anomalen Trichromaten (extrem gelbsichtigen oder blau. sichtigen nach Hering) benutzt. J. v. Kries ${ }^{4}$ ) gibt an, „daß jede ganz beliebige Mischung von Lichtern, deren Wellenlänge mehr als $540 \mu \mu$ beträgt, irgendeinem Gemenge dieses Iichtes mit äußerstem Rot und auch irgendeinem einfachen Licht einer Wellenlänge $>540 \mu \mu$ gleich erscheint". Weiter heißt es dann ${ }^{5}$ ): ,"Man kann also durch Mischung dieser drei Lichter (rotes, grünes und violettes Licht) alle überhaupt vorkommenden Reizarten nahezu vollstänaig erhalten; ganz vollständig allerdings nicht, weil die Umrißlinie der Farbentafel gekrümmt ist (d. h. weil die Mischungen aus Grün und Violett an Sättigung hinter dem homogenen Grünblau zurückbleiben usw.). ${ }^{65}$ Demgemä:B gilt die Gleichung $670 \mu \mu+550 \mu \mu$, die $v$. Kries öfter verwendet ${ }^{6}$ ), als für ihn vollständig. Von Nagel ${ }^{7}$ ) stammt das auf die Gleichung $m L 670+n L 536=o L 589$ eingerichtete Anomaloskop. In der Beschreibung des Apparates erwähnt Nagel beiläufig, es könne ,die Gleichung vollständig gemacht werden (bis auf einen minimalen Sätttigungsunterschied, der wenig anffällig ist)".

1) E. Hering, Individuelle Verschiedenheiten des Farbensinns. Lotos, Neue Folge 6, 37. Prag 1885. Vgl, auch A. Tschermak, Ergebn. d. Physiol. I (2), spez. S. 745. 1903.

2) J. v. Kries, Die Gesichtsempfindung. Nagels Hanđ̋. 3, 110ff. 1905 und auch bereits $M$. v. Frey und $J . v$. Kries, Über die Mischung von Spektralfarben. Du Bois Arch. 1881, S. $336 \mathrm{ff}$.

3) W. A. Nagel, Zwei Apparate für die augenärztliche Funktionsprüfung. Zeitschr. f. Augenheilk. 41, 201. 1907.

4) J. v. Kries, a. a. O. S. 115.

5) J. v. Kries, a. a. O. S. 117.

${ }^{6}$ J. v. Kries, a. a. O. S. 125.

7) W. A. Nagel, a. a. O. speziell S. 217. 
- Kohlrausch ${ }^{1}$ ) benützt die bloßen Tongleichungen $m L 660+n L 540=o L_{h}$ $(660$ bis 540$)$ sogar zur Untersuchung der Summierbarkeit der Flimmer- bzw. Helligkeitswerte.

Allerdings gibt unter anderem $\bar{K} \ddot{\left.o l l n e r^{2}\right)} \mathrm{zu}$, daß für eine Anzahl von Beobachtern die Rayleigh-Gleichung nicht gelingt; das Rot-Grüngemisch bleibt für sie immer etwas ungesättigter gelb wie das $N a$-Licht $\left.{ }^{3}\right)$. ,,Man wählt deswegen die Wellenlängendifferenz kleiner und bedient sich besser anstatt des Thaliumlichtes eines noch etwas mehr gelblichen (also langwelligeren) Grüns."

Was die kurzwellige Hälfte des Spektrums anlangt, sind die Meinungen viel gleichartiger, daß aus Grün und Violett keine vollständige Ton- und Sättigungsgleichung mit Blau gelingt.

Nach diesen Befunden sind jedenfalls die sog. Rayleigh-Gleichung, noch mehr ihre Erweiterungen in Form von Gleichungen zwischen einem reingelben oder schwach grünlichgelben Spektrallicht und einem binären Rot-Grüngemisch oder gar die Gleichung zwischen einer blauen Homogenstrahlung und einem Grün-Violettgemisch ${ }^{4}$ ) - zum mindesten für viele Beobachter - als bloße Farbentongleichungen, nicht als Gleichungen von vollständigem Geltungsgrad zu bezeichnen. Speziell scheint diese Beschränkung für den als Blausichtigkeit (im Sinne E. Herings) bezeichneten Typus der Farbentüchtigen zu gelten - wodurch ein weiteres Charakteristicum für diese so interessante Typenscheidung gegeben zu sein scheint ${ }^{5}$ ). Hingegen ist die Beschränkung für Gelbsichtige weniger weitreichend, ja sie mag in extremen Fällen recht eng sein oder gar nahezu fehlen.

Ungeachtet der anscheinenden prinzipiellen Verschiedenheiten der beiden Typen der Farbentüchtigen, ebenso der Rotgrünblinden bezüglich solcher Gleichungen können doch einerseits die Variationskurven beider Typen - ausgedrückt durch die Komponentenrelation $\frac{X}{Y}-$ teilweise übereinandergreifen; andererseits mag gerade die Unvollkommenheit der Rayleigh-Gleichung in strenger oder ,erweiterter" Form als bloßer Tongleichung ihre praktisch-diagnostische Verwertbarkeit zur Typenscheidung stark einschränken ${ }^{6}$ ). Der eine Beob-

1) A. Kohlrausch, Der Flimmerwert von Lichtermischungen (Tagung d. Dtsch. physiol. Ges. Hamburg 1920). Ber. üb. d. ges. Physiol. u. exp. Pharmakol. 3, 589. 1920.

2) H. Köllner, Störungen des Farbensinns. Karger, Berlin 1912, speziell S. 15. Vgl. auch: Zur Analyse der Rayleighgleichungen der anomalen Trichromaten. Arch. f. Augenheilk. 84, 177. 1919.

3) Typus II am Anomaloskop. Stargardt und Orloff (Diagnostik der Farbensinnstörungen, Springer, Berlin 1912, S. 22) sagen vom Anomaloskop geradezu allgemein: „Bei einer bestimmten Stelle erscheint das obere (gemischte) Feld dem Normalen farblos (!) resp. in einem gewissen gelblichen Tone."

4) Am Anomaloskop Type B. Siehe Nagel ,Zwei Apparate usw." ·

$\left.{ }^{5}\right)$ Vgı. A. Tschermak, Ergebn. d. Physiol. 1 (2), 743. 1902; speziell S. 747.

$\left.{ }^{6}\right)$ Vgl. das kritische Urteil $H$. Köllnes, a. a. O, und Arch. f. Augenheilk. r8. 302. 1915 . 
achter wird trotz der restierenden Sättigungsdifferenz auf Farbentongleichheit einstellen, der andere hingegen nach Helligkeit- bzw. Nuancengleichheit streben und dabei eine Tondifferenz in Kauf nehmen. Vielfach erklärt sich der minder geschulte Beobachter zwar unbefriedigt von der dargebotenen Gleichung, vermag aber den Grund der nichtbeseitigbaren Unvollkommenheit empfindungsanalytisch nicht sicher oder überhaupt nicht aufzuklären, - bald wird er auf reine Sättigungsdifferenz ,tippen“" bald einen bloßen Nüancenunterschied angeben oder vor allem eine Differenz an Gesamthelligkeit behaupten, die er vergeblich durch Änderung der Lichtintensität der einen Gleichungshäifte zu korrigieren suchen wird ${ }^{1}$ ).

Die Differenz der beiden Hälften einer Farbentongleichung zwischen einem homogenen Licht und einem Binärgemisch ist, wie gesagt, zwar nicht durch Intensitätsänderung zu beseitigen, wohl aber durch $\mathrm{Zu}$ mischung von gemischtem bzw. unzerlegtem weißen Licht zur Binärhälfte. Dadurch kann eine Gleichung von vollständigem Geltungsgrad hergestellt werden, deren Hälften nicht bloß an Farbenton, sondern auch an Sättigung und Nuance sowie an Gesamthelligkeit völlig übereinstimmen. Es gilt nun jedesmal eine Reihe von homogenen Lichtern mit je einer Art von Binärgemisch gleich zu setzen, und zwar unter Variation der Intensitätsrelation beider Komponenten sowie der Gesamtintensität des Gemisches und unter abgestufter Zumischung von weißem Licht. Durch die bei dieser Zumischung ermittelten Intensitätsstufen ergibt sich eine messende Charakterisierung - nicht eine wahre Messung - für die Verteilung der Sättigung im untersuchten Spektrumbezirk, welche tabellarisch dargestellt werden wird. Von speziellem Interesse - zumal mit Rücksicht auf die Farbentheorie ist die Frage nach der Lage und Konstanz der empirischen Sättigungsmaxima im Speltrum, sowie nach dem Verhältnis, welches diese durch relative Sättigung ausgezeichneten Punkte im Spektrum erkennen lassen $z u$ den durch tonreine Empfindungseffekte ausgezeichneten Kardinalpunkten (Urgelb, Urgrün, Urblau).

A. Tschermak, der sich selbst bereits seit längerer Zeit mit dieser Frage befaßt hatte, machte mir nun den Vorschlag, dieses Problem systematisch zu bearbeiten.

Auf die farbentheoretische Bedeutung solcher messender Untersuchungen über den Geltungsgrad bzw. über die Sättigungsunterschiede an farbigen Gleichungen, hergestellt zwischen einem spektralen Binärgemisch und der Zwischenreihe homogener Lichter, sei noch durch folgende mathematische Darlegungen vorbereitet.

Sind drei „passend" gewählte Lichter von variabler Stärke gegeben, so ist ein dreidimensionales System der Reize gegeben, welchem nach der oben ent-

I) Siehe H. Aubert, Physiologie der Netzhaut. Breslan 1865, speziell S. 144. 
wickelten Auffassung ein dreidimensionales, jedoch begrenztes Empfindungssystem - versinnbildet durch eine Doppelpyramide - - entspricht. Jeder weitere Dimensionszuwachs des Reizes schafft keinen Dimensionszuwachs der Empfindung mehr, ändert nur die Umrisse eines Nuance- oder Helligkeitsquerschnittes, der bei einem dreidimensionalen Reiz im allgemeinen ${ }^{1}$ ) ein Dreieck, bei einem vierdimensionalen ein Viereck usw. ist. Jetzt erhebt sich die Frage: Nähert sich die Grundfläche einer solchen Pyramide einem Kreis, wenn die Reizreihe eine unendliche Mannigfaltigkeit wird, oder ist ihre Gestalt auch dann ein Polygon von endlicher Seitenanzahl? Anders formuliert: scheint ein Zuwachs an Dimensionen des Reizes bei richtiger Wahl der Einzelkomponenten das System nicht mehr zu ändern? Oder, wenn man als vollkommenen Geltungsgrad einer Gleichung die völlige Effektgleichheit einer Mischung und eines Homogenlichtes bezeichnet: gelingt es mit einer endlichen Komponentenzahl denselben Geltungsgrad zu erreichen wie mit einer unendlichen? Oder, haben alle Gleichungen vollkommenen Geltungsgrades die Form $m \cdot a+n \cdot b=o \cdot c+p \cdot d$ oder gibt es auch Gruppen von Gleichungen vollkommenen Geltungsgrades von der Form: $m \cdot a+n \cdot b=$ $p \cdot d$, wobei $a, b, c, d$ Homogenlichter, $m, n, o, p$ ihre Intensitäten bedeuten?

Wir können das Problem auch von einer anderen Seite anfassen. Hat man eine Reizreihe gegeben, so kann man die ihr entsprechende Empfindungsreihe untersuchen. Man kann aber nur Symbole, am besten graphische Darstellungen in Beziehung setzen. Dabei wird eine zahlenmäßige Charakterisierung der Empfindungen gewisse allgemeine Beziehungen der Empfindungsreihe aufdecken. Außer den Feststellungen, welche die allgemeine Anordnung im Empfindungskoordinatensystem betreffen, ist es wichtig, Transformationen einer gegebenen einfachen Reihe des Reizkoordinatensystems in das der Empfindungen zu kennen. Das scheint prinzipiell mit Hilfe der zahlenmäßigen Charakteristik möglich, doch ergeben sich folgende Bedenken:

Wenn man willkürlich einer Anzahl von Empfindungen Größen zuordnet, so bekommt man je nach der Wahl eine ganze Schar möglicher, durch Projektion ineinander überführbarer Kurven. Zur genauen Bestimmung sind viele Werte nötig, die man aus zahlreichen Untersuchungen ableiten muß, und alle diese müssen eine Figur bilden helfen; das geht nur mit der Fiktion eines konstanten Substrates, die man zwar möglichst anzunähern sucht, deren man sich aber bei der lebenden Substanz nur mit Vorsicht und Vorbehalten bedienen darf. Dies alles zeigt schon, daß so Eigentümlichkeiten der Kurve, wenn sie nicht besonders deutlich sind, dem Untersucher entgehen können.

Hat man sich nun für die Reizreihe der Spektrallichter entschieden, so lassen sie sich allgemein nach Wellenlänge und Intensität der Strahlung darstellen. Alle Lichter eines Prismen- oder Gitterspektrums liegen auf einer Kurve. Alle Empfindungen müssen auch auf einer Kurve darstellbar sein. Bei Mischung der Spektrallichter ergibt sich nun die oben erwähnte Einschränkung, daßß bei unendlicher Mannigfaltigkeit physikalischer Reizmischung und -variation, in der Empfindung neu nur Weiß und Purpur, sonst aber nur Änderungen innerhalb der gegebenen Reihe der Farbentöne, sowie Änderungen an Sättigung und Nüance derart auftreten, daß sie sich symbolisch auf einer Ebene, der Valenzebene, zur Darstellung bringen lassen, und zwar auf einer Fläche, deren Begrenzung die Orte der Homogenlichter und die Purpurstrecke bilden.

Sind auf einer Valenztafel die Orte der Homogenlichter mit ihrer Wellenlängen und Intensitäten eingetragen, so stellt sie, wenn das Sehorgan seinen Zustand nicht

1) „Im allgemeinen" deshalb, weil für den Fall, daß unsere untersuchte Fläche die Grundfläche des Doppelkegels schneidet, Ecken besonderer Art auftreten können. 
ändert, gleichzeitig ein Empfindungstableau dar: eine Schnittfläche des dreidimensionalen Empfindungssystems. Ändert in gewissen Grenzen das Sehorgan seine Stimmung, so bleibt die Valenztafel erhalten, entspricht aber jetzt einem anderen Empfindungstableau, einem anderen Schnitt des Systems. Jede dieser Flächen ist in sich charakterisiert durch Farbenton und Sättigung. Es geht also unter der Fiktion von Konstanz des Substrates, beispielsweise von sog. „,neutraler Stimmung ${ }^{c 6}$, tatsächlich die Valenztafe] in eine Farbentafel über, vorausgesetzt, daß die Orte der Homogenlichter einzeln nach Wellenlänge und Intensität bezeichnet sind. Dann, aber auch nur dann kommen die Bedenken, welche E. Hering äußert'1), in Wegfall.

Das Gesetz der Anordnung auf der Valenztafel ist das Newtonsche Mischungsgesetz. Die physiologische Problemstellung bei seiner Ermittlung ist folgende.

Die betrachteten Farben sind - von der Nuance abgesehen - durch zwei Variable bestimmt: Farbton und Sättigung; sie sind also auf einer Ebene darstellbar. Es bleibt das Gesetz der Anordnung auf dieser Ebene zu suchen. Dasselbe ist dann gefunden, wenn aus drei willkürlich irgendwelchen möglichst verschiedenen Farben zugeordneten Punkten, die nicht auf einer Geraden liegen dürfen, alle den anderen Farben zugeordneten Punkte rechnexisch ableitbar sind; denn drei Punkte bestimmen eine Ebene vollständig. Daß dies tatsächlich für das Newtonsche Mischungsgesetz gilt, haben Maxwell, Helmholtz und König erwiesen. Da es die Mischbarkeit aller auf der Fläche der Farbtafel liegenden Farben aus den Spektralfarben besagt, so ist die nächste Frage die, ob die Spektralfarben aus einer geringen Zahl gemischt erhalten werden können, was auf die Frage hinausläuft, ob der Linienzug von Spektralfarben + Purpur nicht ein einfaches Polygon ist $\left.{ }^{2}\right)$. Aussagen über Farbengrundempfindungen in diesem Sinne lassen sich experimentell machen, ohne andere Voraussetzungen als das Mischungsgesetz. Man hat dabei gar nicht notwendig, irgendwelche Größen einzuführen und vermeidet auch dadurch, daß sich die Untersuchung aus kürzeren Einzeluntersuchungen zusammensetzt, nach Möglichkeit die oben angeführten Fehlerquellen.

Die Methode beruht auf folgender Überlegung: Nimmt man $n$ Urvalenzen an, so liegen alle Farbtöne auf der Peripherie eines Polygons, das mindestens ein Dreieck, im Grenzfall ein Kreis ist. Nimmt man einen Kreis an, in dessen Mittel(Schwer-) Punkt das Weiß zu setzen ist, so heißt das, daß jede Farbe als Ergebnis gleichartiger physiologischer Prozesse aufzufassen ist, keine endliche Anzahl von Urvalenzen und damit auch von deren Indikatoren, von Grundempfindungen existiert. Zieht man in diesem Kreise zwischen zwei Peripheriepunkten eine Sehne, so liegen auf ihr alle aus beiden Endpunkten mischbaren Töne. Sie müssen immer ungesättigter sein als die Empfindung der zugehörigen, auf der Peripherie liegenden Homogenlichter. Am größten wird die Sättigungsdifferenz an dem Orte des arithmetischen Mittels beider Mischungskomponenten sein. Zieht man (vgl, Abb. 1) in einen Kreis $n$ Sehnen von gleicher Länge und läßt $n$ bis ins Unendliche wachsen, untersucht dann die Sättigungsdifferenzkurve zwischen.,Peripherieempfindung " und ,Sehnenempfindang “, so'dürfen, falls es sich um einen Kreis handelt, für $n=\infty$

1) E. Hering, Newtons Gesetz, und zwar S. 6C.

2) Es wurde hier ein merkwürdig scheinender Weg genommen. nämlich von der Voranssetzung ausgegangen, die oben gemacht war: alles, was an Eigenschaften des Effektes richt aus denen des Reizes ableitbar ist, ist im Substrat, an dem die Wirkung auftritt, begründet. Ist die Lichtwirkung im Sehorgan auf „Urvalenzen “ verteilt, so müssen diese, ganz abgesehen davon, ob es gesättigtere Farben als die Spektralfarben gibt, auf unserer Kurve hervortreten. 
keine „Häufungspunkte“ des Sättigungsdifferenzmaximums auftreten, d. h. alle Punkte der Peripherie müssen einmal, besser gleich oft, Maximum der Sättigungs-

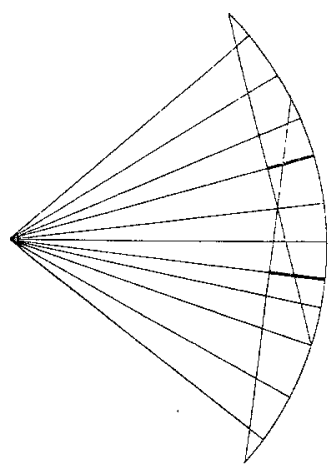

Abb. 1 . differenz gewesen sein. Findet man an einer Stelle einen Häufungspunkt, so sagt das aus, daß an dieser Stelle eine starke Krümmung der Kurve statthat - im Extrem, daB dort eine Ecke ist. Wir wollen diesen Fall untersuchen:

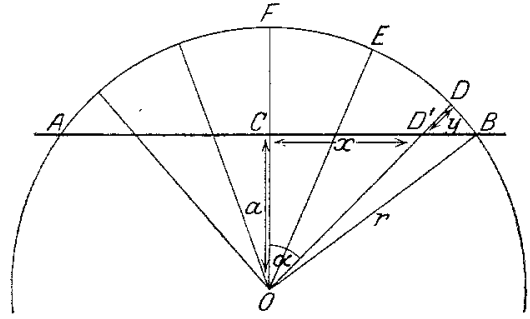

Abb. 2.

1. Es sei (vgl. Abb. 2) $O$ der Mittelpunkt eines Kreises, $\overline{A B}$ eine Sehne, $O D$, $\overline{O E}, \overline{O F}$ usw. seien Radien, von denen durch die Sehne $\overline{A B}$ Stücke abgeschnitten werden.

$$
\begin{gathered}
\overline{O C} \perp \overline{A B} \quad \overline{O C}=a, \quad \overline{O B}=r=\overline{O D}, \quad \bar{C} \overline{D^{\prime}}=x, \quad \overline{D D^{\prime}}=y . \\
\sin \alpha=\frac{\overline{C D^{\prime}}}{\overline{O D^{\prime}}}=\frac{x}{r-y}, \\
\operatorname{tg} \alpha=\frac{\overline{C D^{\prime}}}{\overline{C O}}=\frac{x}{a},
\end{gathered}
$$

nun ist

$$
\sin \alpha=\frac{\operatorname{tg} \alpha}{\sqrt{1+\operatorname{tg}^{2} \alpha}} \quad \begin{aligned}
& \text { (goniometrische Form des } \\
& \text { pythagoreischen Lehrsatzes) }
\end{aligned}
$$

also

$$
\frac{x}{r-y}=\frac{\frac{x}{a}}{\sqrt{1+\left(\frac{x}{a}\right)^{2}}}=\frac{x}{\sqrt{a^{2}+x^{2}}},
$$

daraus $\quad(r-y)^{2}=a^{2}+x^{2}, \quad$ (a $y<r$ sein muß. $y=r-\sqrt{a^{2}+} \overline{x^{2}}$,

$$
(y-r) d y=x d x
$$

$$
\frac{d y}{d x}=\frac{x}{\sqrt{a^{2}+x^{\overline{2}}}},
$$

für:

$$
x=0: \quad y=r-a, \quad \frac{d y}{d x}=0, \quad \frac{d^{2} y}{d x^{2}}<0 .
$$

$y$ also ein Maximum, was wir schon oben für den Kreis sagten.

2. Man kann nun um jede Ecke vom Schwerpunkt einesVieleckes einen Kreis legen (Abb. 3). Dann gilt folgendes:

Es sei, wie früher, $\overline{A B}$ eine Sehne, ihr Normalabstand von $O$ wieder $a$. Die Sehne $\overline{B C}$ habe den Normalabstand $b, b>a$.

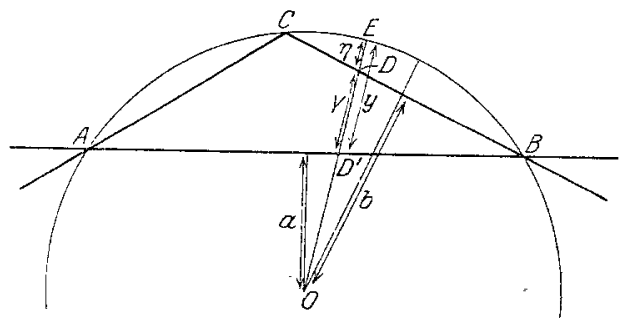

Abb. 3. 
Nach 1. ist für $\overline{A B}$ :

$$
\begin{aligned}
(r-y)^{2} & =a^{2}+x^{2}, \\
(r-\eta)^{2} & =b^{2}+\xi^{2} . \\
I^{+}=\overline{D^{\prime} D} & =\overline{D^{\prime} E}-\overline{D E} \\
& =y-\eta .
\end{aligned}
$$

Zeichnen wir also Kurve (1) und Kurve (2) so über derselben $X$-Achse, daß3 beide im Punkte $B\left(y=\eta=0, x=\sqrt{r^{2}-a^{2}}\right)$ die $X$-Achse schneiden und drücken wir $\xi$ durch $x$ aus:

$$
\begin{aligned}
\varsigma & =x+\sqrt{r^{2}-b^{2}}-\sqrt{r^{2}-a^{2}}, \quad\left|\overline{r^{2}-a^{2}}=p, \quad\right| \overline{r^{2}}-b^{2}=q, \\
& =x+q-p,
\end{aligned}
$$

in 2 eingesetzt: $\quad(r-\eta)^{2}=b^{2}+(x+q-p)^{2}$,

aus 1

$$
y=r-\sqrt{a^{2}+x^{2}}
$$

aus 2 a

$$
\eta=r-\sqrt{b^{2}+(x+q-p)^{2}},
$$

nach 3 ist

$$
\begin{aligned}
Y & =y-\eta=\sqrt{b^{2}+(x+q-p)^{2}-\left(\overline{a^{2}}+x^{2}\right.} \\
d Y & =\frac{x+q-p}{\sqrt{b^{2}+(x+q-p)^{2}}}-\frac{x}{\sqrt{a^{2}}+\overline{x^{2}}} .
\end{aligned}
$$

In Punkte $C$, unserer Eeke ist nun, was aus 2 a folgt: $\quad r^{2}=b^{2}+(x+q-p)^{2}$,

$$
\begin{aligned}
r^{2}-b^{2} & =(x+q-p)^{2}, \quad r^{2}-b^{2}=q^{2}, \\
\pm q & =x+q-p, \\
x_{1} & =p=\sqrt{r^{2}-a^{2}} \quad(\text { Abszisse von B), } \\
x_{2} & =p-2 q, \\
x & =p-2 q, \\
a Y & =\frac{-q}{\sqrt{b^{2}+q^{2}}}-\frac{p-2 q}{\sqrt{a^{2}+(p-2)^{2}}}, \quad b^{2}+q^{2}=r^{2} \\
\overline{d x} & =-\left(\frac{q}{r}+\frac{p-2 q}{\sqrt{a^{2}+(p-2 q)^{2}}}\right) .
\end{aligned}
$$

für $C$ ist also

Ist $C$ ein Grenzmaximum, so $m u \frac{d Y}{d x}<0$ sein, da $b>a>0$ ist, genügt es für den Differentialquotienten $\frac{d Y}{d x}$ die beiden Werte zu ermitteln, die er annimmt, wenn man $a=0$ und dann gleich $b$ setzt, also die Grenzen bestimmt, innerhalb derer $\frac{d Y}{d x}$ variieren kann:

$$
\begin{aligned}
& \text { für } a=0 \text { wird } \frac{d Y}{d x}=-\left(\frac{q}{r}+\frac{r-2 q}{r-2 q}\right)=-\left(\frac{q}{r}+1\right), \text { also }<0 \\
& \text { für } a=b, \text { wird } p=2 \begin{aligned}
\frac{d Y}{d x} & =-\frac{q}{r}+\frac{q}{r}, \quad \text { da ja } \sqrt{b^{2}+q^{2}}=r, \\
& =0, \\
& \begin{aligned}
d Y \\
d x
\end{aligned}
\end{aligned}
\end{aligned}
$$

folglich ist für $a<b$ :

d. h. also: $C$ ist immer ein Grenzmaximum, da $b$ in unserem Falle immer größer ist als $a$.

Es ist nun (vgl. Abb. 4) sofort klar, daß, was für $A B C$ gilt, noch viel mehr für $A^{\prime} C B^{\prime}$ gilt. Das heißt: Legt man vom Schwerpunkt $O$ aus um einen Eckpunkt einen Kreis, schneidet dann die Ecke durch eine Gerade (Mischlinie) ab, so ist die 
Ecke ein Differenzmaximum in unserem Sinne, wenn die Gerade die Seiten der Ecke innerhalb des umschriebenen Kreises schneidet.

Ist dies nicht der Fall (vgl. Abb. 5), dann schneidet $A B$ die Seite der Ecke in $B^{\prime}$ außerhalb der Kreisperipherie. Das Extrem ist erreicht, wenn $B$ im Un. endlichen liegt, dann gilt

oder

$$
\begin{aligned}
x & =\xi \\
d Y & =\frac{x}{\sqrt{b^{2}+x^{2}}}-\frac{x}{\sqrt{a^{2}+x^{2}}} \\
d x & \quad \text { für }: \frac{x}{\sqrt{x^{2}+b^{2}}}<\frac{x}{\sqrt{x^{2}+a^{2}}} \\
\frac{d Y}{d x}<0, \quad & >b^{2}+b^{2} \\
b & >a .
\end{aligned}
$$

Was hier in drei Fällen für die Seite $C B$ entwickelt wurde, gilt natürlich auch für $C A$. Es ist also $C$ auf jeden Fall ein Maximum, das am besten hervortreten wird, wenn man den ersten Fall (Abb. 3 oder 4) wählt.

Hat also unsere untersuchte Kurve deutliche Häufungsmaxima und läßt sich weiterhin jeststellen, daß zwei solche benachbarte Punkte miteinander durch Gerade

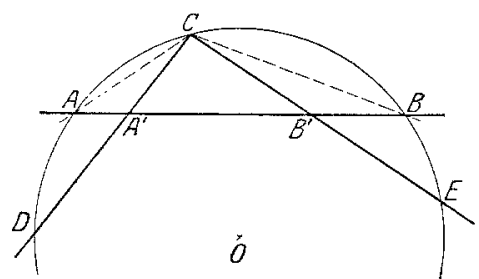

Abb. 4.

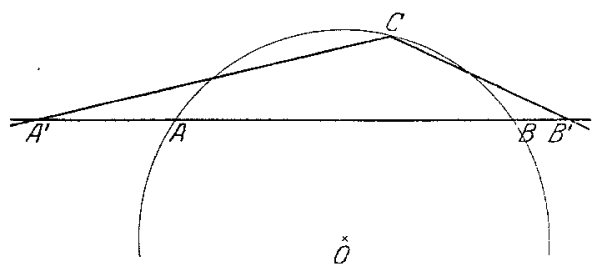

Abb. 5.

verbunden sind, d. h. alle zwischen beiden liegenden Farben aus diesen vollständig ohne Sättigungsdifferenz erhalten werden kömnen, so verhält sich die Kurve, als ob sie an solchen Stellen Ecken hätte. Hat man in der Farbenebene $v$ Punkte und verbindet $n$ Punkte derart, daß ein geschlossenes $N$-Eck entsteht, ohne irgendwelche erhabene Winkel, und alle $v$ Punkte innerhalb oder auf der Peripherie des geschlossenen $n$-Ecks liegen, dann sagt das Schwerpunktsgesetz aus, daß jegliche Farbe, deren Ort einem Punkte innerhalb des vom $n$-Eck umschlossenen „Farbenfeldes" entspricht, als aus den $n$ Farben der Ecken gemischt gedacht werden kann.

Ist das Farbensättigungsfeld ein Kreis, so heißt das also, daß man, um tatsächlich aus den Farben, deren geometrischen Ort die Peripherie darstellt, alle Punkte dieses Feldes erhalten zu können, alle unendlich vielen Peripheriepunkte als gegeben annehmen muB, da ja ein Kreis als Vieleck von unendlich vielen Seiten - somit auch Ecken - betrachtet werden kann. Es ist also dann überhaupt nicht statthaft, alle Punkte des Feldes als aus einer endlichen Anzahl von Grundpunkten konstruiert zu denken. Nur im Falle, daß Eckpunkte konstatiert werden können, ist man berechtigt, soviel Grundpunkte anzunehmen, als Ecken vorhanden sind. Da physikalisch im Speltrum keine Ursache für das Auftreten speziell bevorzugter Punkte gegeben is ${ }^{1}$ ), so muß diese Eigentümlichleit eine physiologische sein. Sie muß sich auch in der reellen Farbentafel, die von der Kurve der Spektralfarben + Purpur umschlossen ist, äußern. Man kann dies auch so sagen: da die theoretische Farben-

1) Das Strahlungsmaximum liegt für das Prismenspektrum der gewöhnlich verwendeten Lichtquellen im Ultrarot. 
tafel ein Punktsystem nach Art eines Flächengitters darstellt, muß auch die Kurve der Spektralfarben dieser Struktur entsprechen.

Mit diesen Ausführungen ist auch schon das Untersuchungsgebiet gegeben. Man muß Reihen von Gleichungen vollkommenen Geltungsgrades herstellen.

\section{Methodit.}

Die Untersuchungen wurden an dem im Prager Institut vorhandenen, ursprünglichen Heringsehen Spektrallichtermischapparat mit gewöhnlichem, nicht polarisiertem Licht angestellt, einem Apparat, welcher seinerzeit zum Teil aus Bestandteilen vorhandener Spektralapparate unter Mitwirkung von Lippich gebaut worden war. Ein vervollkommneter Apparat dieser Art, ein wahres Universalinstrument, befindet sich bekanntlich im Leipziger Institut; dieser wurde unlängst von Garten ${ }^{1}$ ) genau beschrieben.

Der Prager Apparat ist primitiver: er besteht (vgl. Abb. 6) aus zwei Spektralapparaten $A$ und $B$, welche Spektren auf die Vorderspalte $S_{1}$ bzw. $S_{2}$ entwerfen, die daraus einen schmalen Bereich ausschneiden. Diese Strahlung gelangt auf der Seite von $B$ zur Linse $L_{1}$ und zum Fernrohr $F$; für $A$ wird dies durch totale Reflexion an dem dreiseitigen Prisma Pr erreicht. Der Beschauer stellt das Fernrohr auf die Kante $V$ von $P r$ scharf ein, sieht also durch diese getrennt - - im umgekehrten Bilde - zwei farbige Feldhälften, die rechte von $B$, die linke von $A$ geliefert. Die Größe des Feldes wird durch eine Irisblende hei $J$ geregelt. Als Fixationspunkt dient eine geeignete Kerbe der Prismenkante. Ber $C$ befindet sich ein Stutzen mit Spalt zum Zulassen von weißem Licht, aas durch die Solinglasplatte $G$ der von $B$ kommenden farbigen Strablung zugespiegelt werden kann. Die Menge des weißen Lichtes wird durch die Weite dieses Spaltes $\left(S_{3}\right)$ reguliert. Die Spalte $S_{1}$ und $S_{\mathrm{g}}$ gestatten die Intensitäten der von $A$ und $B$ kommenden Strahlungen zu variieren. $H S$ ist ein horizontaler Spalt, welcher die Intensität beider Feldhälften gleichmäßig zu ändern gestattet, - soweit dazu nicht ein zwisehen der Linse $L$ und diesem Spalte einschaltbarer Episkotister mit gegen Seitenlicht dichter Hülse verwendet wird; doch blieben beide Abstufungseinrichtungen für die vorliegende Untersuchung unbenützt. - Sowohl am Kollimator $A$ wie am Kollimator $B$ kann man einfache oder gekoppelte Doppelspalte anbringen, wobei die Scheidung der beiden Ausschnitte durch je ein Einsatzstück aus einer unter starken Sprüngen abgestuften Breitenserie bewerkstelligt wird, während am Leipziger Apparate durch ein sog. Harmonikaschaltstück eine kontinuierliche Abstufung möglich ist. Wir konnten demgemäß nur Binärgemische von ganz bestimmten, nicht von beliebigen Wellenlängenunterschieden verwenden.

Um den Apparat zu eichen, hat A. Tschermak eine praktische Einrichtung angebracht: $S_{1}$ und $S_{2}$ haben von $L_{1}$ gleichen Abstand. $L_{1}$ entwirft von ihnen reelle Bilder, die man mit dem Fernrohr, dessen Okular weit genug ausgezogen werden kann, betrachtet. $S_{1}$ und $S_{2}$ sind nun die Orte der durch $A$ und $B$ entworfenen Spektra. Es genügt also einfach das Ausziehen des ganzen Fernrohrs bis zur genauen Einstellung von $S_{1}$ und $S_{2}$, um scharfe Bilder der Fraunhoferschen Linien, oder, was sich sehr gut bewährt hat, nach dem Vorgange von $A$. Tschermak ${ }^{2}$ )

1) S. Garten, Herings Farbenmischapparat für spektrale Lichter. Zeitschr. f. Biol. 12, 89. 1920. Vgl. auch C. v. Hess, Methoden zur messenden Untersuchung des Licht- und Farbensinnes sowie des Pupillenspieles. Abderhaldens Handbuch der biologischen Arbeitsmethoden. Abt. V. Bd. VI, S. 159 ff. 1921.

2) A. Tschermak, Über spektrometrische Verwendung von Helium. Pflügers Arch. f. d. ges. Physiol 88. 95. 1901). Die Kapillarstrecke des mit Helium gefüllten Plückerrohres läßt sich mittels einer geeigneten TrägerhüIse bequem vor die einzelnen Kollimatorspalte bringen. 
Bilder dex Emissionslinien von Helium zu erhalten; damit kann man schnell und bequem die Eichung durchführen. Die Ablesung des Kollimatorstandes gesehieht an einer Spiegel-Skalenfernrohr-Einrichtung nach Poggendorf; die Ermittlung der

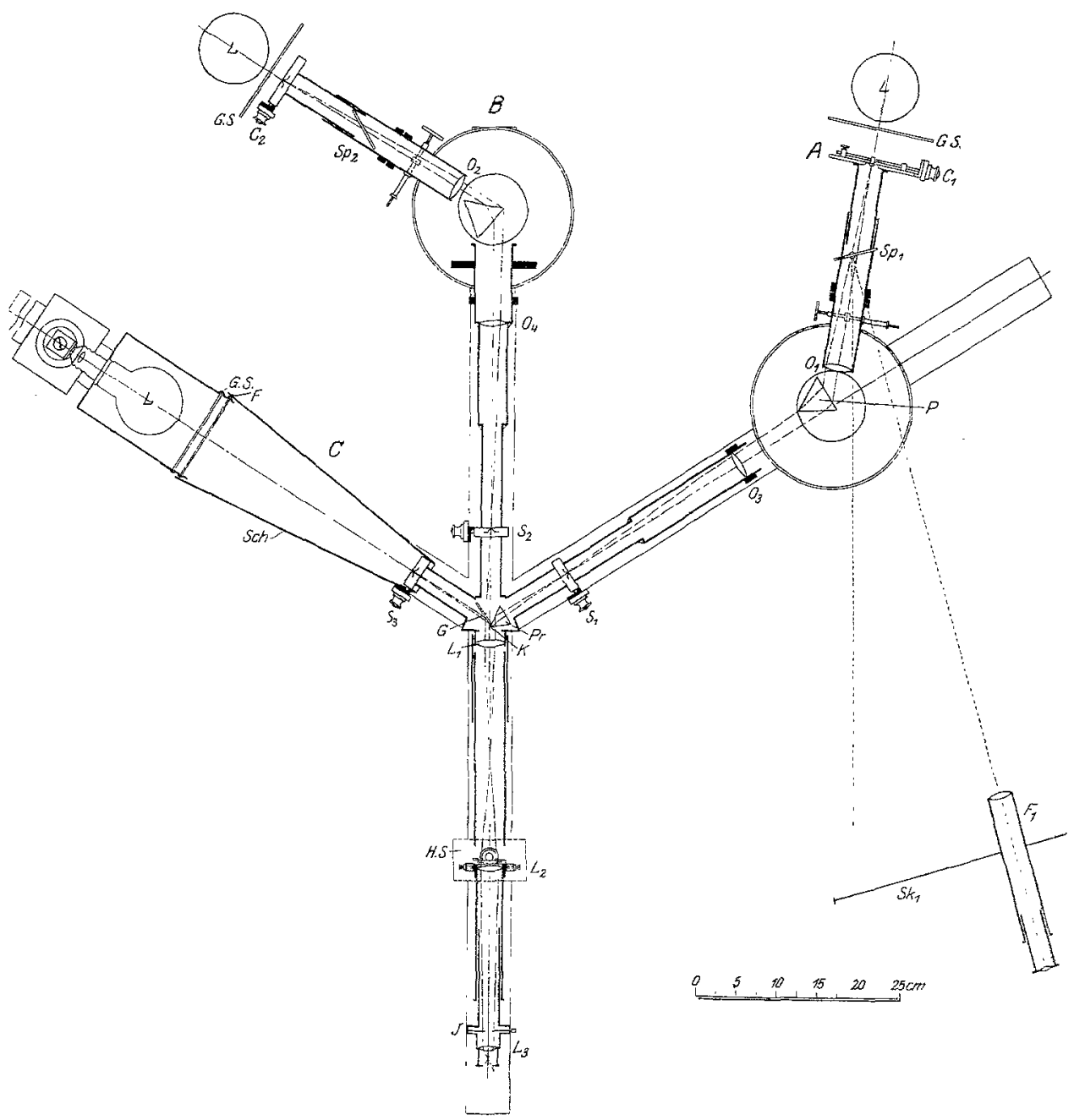

Abb. 6. Apparatkonstanten. $\left(O_{1}, O_{2}\right)$ Kollimatorlinsen: Brennweite je $25 \mathrm{~cm} ;\left(O_{3}, O_{4}\right)$ Okulalspaltrohrlinsen: Brennweite je $25 \mathrm{~cm} . L_{1}$ Vorderlinse $7,5 \mathrm{~cm}$ Brennweite. Fernrohr mit $L_{s}$ Okular 2,5 cm, $L_{2}$ Objektiv 11,2 cm. Abstand: Kollimatorspalt-Kollimatorlinse 25 cm. dbstand : Okularspalt-Vorderlinse $14 \mathrm{~cm}$. Abstand: Fernrohrobjelxtiv-Vorderlinse $27,5 \mathrm{~cm}$. Maximale Feldgröße $15^{\circ}, 5,5 \mathrm{~cm}$ auf $21 \mathrm{~cm}$. 3 Lampen à 100 Kerzen, Tungsram 70 Watt.

zugehörigen Wellenlängen mit Hilfe graphischer Darstellung, für weiche die Heliumliuien $(706,5 ; 667,6 ; 587,6 ; 501,6 ; 492,3 ; 471,3 ; 447,1$, nach neueren Messungen) die Grundpunkte abgeben.

Als Lichtquelle diente das durch Mattierung der Birnen und Vorschaltung einer Mattglasplatte (G.S.) gleichmäßig zerstreute Licht einer Tungsram-Glüh- 
lampe von 100 Kerzen Stärke bzw. 70 Watt als Lichtquelle. Das ergänzende Weißlicht wurde gleichfalls von einer Tungsram-Glühlampe gewonnen unter Filtration des ganz schwach gelblichen Lichtes durch ein unsattblaues Kobaltglas von geeignetem Tinktionsgrade $\left(F^{\prime}\right)$. Die seitliche Lichtzunischung aus dem Beobachtungs. raum war durch einen Schlot aus Pappe (Sch.) zwischen Lampe, Glas und Spalt vermieden. Es sei bereits hier betont, daß selbst für den Fall einer geringen $A b$ weichung des beigemischten Lichtes von „Weiß“" gegen eine Farbe, die zu erwartenden "Eckpunkte" genau so deutlich hervortreten müssen.

Für meine Versuche benützte ich in $A$ einen gekoppelten Doppelspalt. Die Summe beider Spalte war 1,5 mm. An Einsatzstücken wurden solche von 4,5; 3,$5 ; 2,9 ; 2,3 \mathrm{~mm}$ Breite verwendet. Die Breite des Spaltes bei $B$, der homogenes Licht lieferte, blieb während jeder Versuchsreihe konstant; außer bei einer Reihe, bei der dies ausdrücklich vermerkt ist. Seine Breite war: $0,4-0,5 \mathrm{~mm}$ (110 bis 140 Skalenteile). Ebenso blieb $S_{2}$ konstant; $S_{1}$ wurde variiert, die Änderungen wurden aber nicht registriert. Die Anordnung war jedoch so getroffen, daß der Spalt $S_{1}$ immer schmal blieb und meist nur in sehr engen Grenzen variiert wurde. Die Größe des streng zentral fixierten Feldes betrug: $2^{\circ} 30^{\prime}$. Das Bild war demnach kaum größer als der stäbchenfreie Bezirk mit $1^{\circ} 45^{\prime}$ bis $3^{\circ} 30^{\prime}$ Ausdehnung: die Gleichungen sind demnach alszentrale und foveale zu bezeichnen. Der Kopf des Beobachters war durch einen Gebißhalter fixiert. Durchwegs wurde nur das rechte Auge verwendet. Was die Beobachtungstechnik anlangt, so war sie folgende. Es wurden immer Reihen von mindestens 4 Gleichungen gemacht. Dabei wurde zunächst mit der größten Sorgfalt Tongleichheit hergestellt und dann Weiß beigemiseht, wenn sich Sättigungsdifferenzen ergaben. Da es sich zeigte, daß das Verhältnis der Spaltbreiten nach Tagen sich kaum änderte (natürlich innerhalb der Fehlergrenzen), die Weißbeimischung dagegen von Tag zu Tag größeren Schwankungen unterworfen war, wahrscheinlich abhängig von der Stimmung des Auges, so gewöhnte ich mich, erst sorgfältig das Verhältnis der Spaltbreiten und damit Farbentongleichheit zu erreichen, und dann in verhältnismäßig kurzer Zeit (ca. 1 Stunde) die vollständigen Gleichungen zu ermitteln. Man darf nur ganz kurz beobachten, sonst schwinden Sättigungsdifferenzen, woranf besonders E. Hering ${ }^{1}$ ) hinweist. Hat man einmal Gleichheit eingestellt, so muß man einige Minuten warten, dann prüfen, von nevem einstellen und sofort, bis die Gleichheit völlig konstant ist. Dabei ist es manchmal sehr schwierig, mehr auszusagen, als daß beide Feldhälften nicht genau gleich erscheinen, und man muß durch Herumprobieren ansfindig machen, ob es an Farbenton, Sättigung oder Nuance liegt ${ }^{2}$ ). Die Versuche wurden bei guter Helladaptation angestellt.

Anschließend möchte ich meinen Farbensinn charakterisieren. Es fiel mir zunächst vor 4 Jahren auf, daß mir Tuberkelbacillen bei ZiehtNielsen-Färbung nur dunkel auf blauem Grunde, nicht aber rot erschienen. Untersuchungen as Nagels Anomaloskop ergaben, daß ich viel mehr Rot im Gemisch zur Gleichung brauchte als andere mit ,normalem" Farbensinn (Einstellung 67 gegen 56-57). Nach manchen Stillingschen Tafeln könnte man bei mir fast Farbenblindheit, und zwar sog. Rotblindheit, diagnostizieren. Dies kann jedoch durch andere Proben (Wollproben, Heringscher Glaslichterapparat usw.) ausge-

1) E. Hering, Über Newtons Gesetz der Farbenmischung. Lotos N. F. 1, 1877, speziell S. 44 .

7) H. Aubert, Physiologie der Netzhaut. 1865, speziell S. 125. 
schlossen werden, vor allem aber durch Versuche am Spektrallichtermischapparat. Ich führe hier eine Tabelle an, die zeigt, daß meine Abweichung auf physiologische, nicht auf physikalische Verschiedenheiten zurückgeführt werden muß, da - entsprechend der Argumentation von

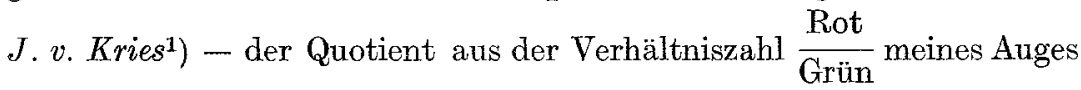
und der Verhältniszahl des ,normalen“ Auges von Dr. M. H. Fischer nicht konstant ist.

$\begin{array}{cccc}\text { Gleichung } 542+652=(20 . \text { II. 1921): } & & \\ \text { Ih } & G & \text { F } & \text { Q } \\ 610 & 3,495 & 1,610 & 2,396 \\ 590 & 1,439 & 0,5901 & 2,439 \\ 580 & 0,944 & 0,366 & 2,581 \\ 570 & 0,584 & 0,246 & 2,371 \\ 560 & 0,510 & 0,178 & 2,865\end{array}$

In der ersten Kolumne stehen die Wellenlängen des homogenen Vergleichslichtes, in der zweiten dieVerhältnisse $\frac{\text { Rot }}{\text { Grün }}$ für den Beobachter G., in der dritten die für Dr. $F$, in der vierten das Verhältnis $\mathrm{Q}=\frac{\mathrm{G}}{\mathrm{F}}$.

Aus all dem ergibt sich, daß es sich bei mir um ein Farbensystem nach Art von jenem des $M r$. Hart ${ }^{2}$ ) und $M$. Levys ${ }^{3}$ ) oder eines extrem Blausichtigen nach Herings Nomenklatur, eines Protanomalen nach $J$. v. Kries handelt. Ich kann im allgemeinen das bestätigen, was Levy beschreibt. Auffallend stark ist meine Kontrastfunktion; ein Umstand, welcher mir beim Einstellen von Gleichungen, was Genauigkeit angeht, sehr zu statten kommt, was hingegen die Zeit anlangt, die zum Einstellen benötigt wird, sehr aufhält, da Sättigungs- und Helligkeitsunterschiede kontrastgebend wirken. Alle diese Erscheinungen treten aber nur für den langwelligen Teil des Spektrums hervor. Die Versuche habe ich an mir als einer geeigneten Versuchsperson angestellt, weil nach den Angaben von $E$. Hering ${ }^{4}$ ) für Dr. $S$., einem relativ stark Blausichtigen, Mischgelb deutlich ungesättigt erschien gegenüber Homogengelb, und auch $A$. Tschermak als in gewissem Grade blausichtig (jedoch weit weniger als ich) für sich deutliche Sättigungsunterschiede bemerkt und zu messen begonnen hatte. Ich habe aber Dr. M. H. Fischer als einen Gelbsichtigen (auch gegenüber $A$. Tschermak) ersucht,

1) J. v. Kries, Über Farbensysteme. Zeitschr. f. Psychol. u. Physiol. d. Sinnesorg. 13, 287. 1897 und Über die anomalen trichromatischen Farbensysteme. Ebenda 19, 63. 1898.

3) Rayleigh, Nature 1. c.

3) Max Levy, Über einen zweiten Typus des anomalen trichromatischen Farbensystems. Inaug.-Diss. Freiburg i. Br. 1903. Vgl. auch H. Köllner, Arch. f. Augenheilk. 84, 177. 1919.

$\left.{ }^{4}\right)$ E. Hering, Über individuelle Verschiedenheiten des Farbensinns, spez. S. 37. 
meine Versuche nachzuprüfen. In den Ergebnissen werde ich diese Resultate mit anführen. Ich möchte gleich an dieser Stelle Herrn $D r . M$. H.Fischer, Assistenten am Institut, für seine Liebenswürdigkeit meinen besten Dank aussprechen.

\section{III. Übersicht der Versuchsergebnisse.}

Trotzdem festgestellt werden konnte, daß von Tag zu Tag manchmal sogar beträchtliche Schwankungen in den absoluten Werten der Weißbeimischung auftraten, ergab es sich doch, daß die allgemeine Kurvenform erhalten blieb. Es sei ein Beispiel hierfür angefübrt.

Tabelle $I$.

Gleichung L $550+\mathrm{L} 468=\mathrm{L} 490$ bis $540 \mu \mu$.

9. XI. 1920.

a
10. XI. 1920 .

Reihe I.

\begin{tabular}{|c|c|c|c|c|c|c|c|}
\hline Lh & $\begin{array}{l}\text { Weißbei- } \\
\text { mischung }\end{array}$ & 550 & 468 & Lh & W. B. & 550 & 468 \\
\hline 490 & 88 & 41 & 429 & 490 & 72 & 51 & 419 \\
\hline 500 & 71 & 109 & 361 & 500 & 53 & 93 & 377 \\
\hline 510 & 197 & 160 & 310 & 510 & 198 & 158 & 312 \\
\hline 520 & 288 & 195 & 275 & 520 & 212 & 213 & 257 \\
\hline 530 & $\mathbf{3 5 3}$ & 243 & 227 & 530 & 316 & 243 & 227 \\
\hline 540 & 184 & 316 & 154 & 540 & 220 & 316 & 154 \\
\hline
\end{tabular}

Gleichung I $564+\mathrm{L} 476=\mathrm{L} 520$ bis $550 \mu \%$.
15. X. 1920 .

Reihe II.

\begin{tabular}{c|c|c|c|c|c|c|c}
\multicolumn{4}{c}{} & \multicolumn{2}{c}{ Reihe II. } & \multicolumn{2}{c}{$\mathrm{b}^{\prime}$} \\
\hline \hline Lh & W. B. & 564 & 476 & Ih & W. B. & 564 & 476 \\
\hline 520 & 758 & 145 & 325 & 519 & 550 & 105 & 355 \\
530 & $\mathbf{8 8 0}$ & 155 & 315 & 529 & $\mathbf{8 0 0}$ & 117 & 343 \\
540 & 732 & 194 & 276 & 539 & 700 & $\mathbf{1 5 5}$ & 305 \\
550 & 550 & 236 & 234 & 549 & 550 & 204 & 256
\end{tabular}

Daraus geht hervor, daß das Maximum sich konstant erhält, trotzdem manche Weißwerte stärkere Schwankungen aufweisen. Dann wurde zur Reihenbeobachtung geschritten. Ich führe hier nur einige Serien von Beobachtungsreihen an:

\section{Tabelle $I I$.}

\section{A. Gelbgleichungen.}

\section{Reihe I.}

a) Gleichung $690+567$. b) Gleichung $670+557$. c) Gleichung $655+547$. 18. IX. 1920 nachmittags. 18. IX. 1920 vormittags. 17. XI. 1921 nachmittags. Einsatz I.

Einsatz I.

Einsatz I.

\begin{tabular}{|c|c|c|c|c|c|c|c|c|c|c|c|}
\hline Lh & W. B. & 690 & 567 & Lh & W. B. & 670 & 557 & Lh & W. B. & 655 & 547 \\
\hline 569 & 0 & 290 & 170 & 55 & 0 & 244 & 210 & 559 & 12 & 87 & 374 \\
\hline 574 & 30 & 335 & 125 & 56 & 13 & 272 & 188 & 569 & 44 & 189 & 271 \\
\hline \multirow{3}{*}{579} & 13 & 378 & 82 & 574 & 15 & 281 & 179 & 574 & 33 & 226 & 234 \\
\hline & & & & 579 & 15 & 31 & 142 & 579 & 24 & 260 & 200 \\
\hline & & & & 589 & 2 & 360 & 100 & 589 & 13 & 289 & 171 \\
\hline
\end{tabular}


Tabelle II (Fortsetzung).

d) Gleichung $643+541$. e) Gleichung $633+535$. f) Gleichung $623+527$. 19. IX. 1920 nachmittags. 20. IX. 1920. Einsatz I. 21. IX. 1920. Einsatz I.

\begin{tabular}{c|r|r|r|c|c|c|c|c|c|c|c}
\hline \hline Lh & W. B. & 643 & 541 & Ih & W. B. & 638 & 585 & Lh & W. B. & 623 & 527 \\
\hline 559 & 0 & 140 & 320 & 559 & 21 & 103 & 357 & 559 & 35 & 83 & 377 \\
569 & $\mathbf{2 2}$ & $\mathbf{1 9 9}$ & $\mathbf{2 6 1}$ & $\mathbf{5 6 9}$ & $\mathbf{4 6}$ & 126 & 334 & 565 & 48 & $\mathbf{1 0 5}$ & 355 \\
574 & 12 & 206 & 254 & 579 & 31 & 189 & 271 & 569 & $\mathbf{7 8}$ & $\mathbf{1 3 1}$ & 329 \\
579 & 8 & 234 & 226 & 589 & 10 & 233 & 227 & 574 & 49 & 154 & 306 \\
589 & 0 & 302 & $\mathbf{1 5 8}$ & & & & & 579 & 39 & 177 & 283 \\
& & & & & & & & 589 & 23 & 218 & 242
\end{tabular}

g) Gleichung $612+521$. h) Gleichung $602+516$. i) Gleichung $592+508$. 22. IX. 1920. Einsatz I. 23. XI. 1920. Einsatz I. 24. IX. 1920. Einsatz I.

\begin{tabular}{l|r|r|r|r|r|r|r|r|r|r|r}
\hline Lh & W. B. & 612 & 521 & Lh & W. B. & 602 & 516 & Lh & W. B. & 592 & 508 \\
\hline \hline 559 & 78 & 75 & 385 & 559 & 88 & 67 & 393 & 559 & 44 & 81 & 379 \\
569 & $\mathbf{1 0 0}$ & 103 & 357 & 564 & 108 & 81 & 379 & 564 & 50 & 91 & 369 \\
579 & 78 & 148 & 312 & 569 & $\mathbf{1 1 7}$ & 89 & 371 & $\mathbf{5 6 9}$ & $\mathbf{5 8}$ & 103 & 357 \\
589 & 30 & 185 & 275 & 574 & 108 & 100 & 360 & 579 & $\mathbf{4 8}$ & 126 & $\mathbf{3 3 4}$ \\
& & & & 579 & 88 & 125 & 335 & 589 & 0 & 280 & $\mathbf{1 8 0}$
\end{tabular}

k) Gleichung 583+502. 25. IX. 1920. Einsatz I').

\begin{tabular}{c|c|c|c}
\hline Lh & W. B. & 583 & 502 \\
\hline 559 & $0(?)$ & 112 & 348 \\
564 & 38 & 130 & 330 \\
569 & 28 & 138 & 322 \\
574 & 23 & 168 & 292 \\
579 & 0 & 252 & 208
\end{tabular}

Reihe II.

$\left.a^{\prime}\right)$ Gleichung $\left.678+534 . \quad b^{\prime}\right)$ Gleichung $\left.664+528 . \quad c^{\prime}\right)$ Gleichung $653+522$. 11. XI. 1920. Einsatz II. 29. IX. 1920. Einsatz II. 1. X. 1920. Einsatz II.

\begin{tabular}{c|r|r|r|r|r|r|r|r|r|r|r|r|r}
\hline \hline Ih & W. B. & 678 & 584 & Lh & Bh $\left.^{2}\right)$ & W. B. & 664 & 528 & Lh & w. B. & 653 & 522 \\
\hline \hline 559 & 40 & 360 & 110 & 559 & 296 & $\mathbf{2 5 0}$ & 281 & $\mathbf{1 7 9}$ & 559 & 706 & 263 & $\mathbf{1 9 7}$ \\
569 & $\mathbf{1 0 0}$ & 384 & 86 & 569 & 210 & 920 & 319 & $\mathbf{1 4 1}$ & 569 & $\mathbf{7 8 0}$ & $\mathbf{3 0 7}$ & $\mathbf{1 5 3}$ \\
$\mathbf{5 7 9}$ & 33 & 397 & 73 & $\mathbf{5 7 4}$ & 196 & $\mathbf{1 0 7 0}$ & 334 & $\mathbf{1 2 6}$ & $\mathbf{5 7 4}$ & $\mathbf{7 4 0}$ & 317 & 143 \\
$\mathbf{5 8 9}$ & 43 & 424 & 46 & 579 & $\mathbf{1 7 5}$ & 390 & $\mathbf{3 4 5}$ & $\mathbf{1 1 5}$ & $\mathbf{5 7 9}$ & 500 & 325 & $\mathbf{1 3 5}$ \\
$\mathbf{5 9 9}$ & 0 & 454 & 36 & 589 & $\mathbf{1 4 9}$ & 0 & $\mathbf{3 8 4}$ & 76 & 589 & $\mathbf{3 0 0}$ & $\mathbf{3 5 3}$ & $\mathbf{1 0 7}$
\end{tabular}

1) In der ersten Kolonne stehen die Vergleichshomogenlichter (Lh), in der zweiten die Weißmischungen (W. B.), in der dritten und vierten die Mengen des Mischungskomponenten in zufälligen Maßeinheiten (Skalenteilen).

2) Weil hier auch die Breite des Homogenspaltes variiert wurde, wird diese hier mit angegeben. 
Tabelle II (Fortsetzung).

d.) Gleichung $643+517,5$. e') Gleichung $633+512$. f $^{\prime}$ ) Gleichung $623+507$.

2. X. 1920. Einsatz II. 3. X. 1920. Homogen- 5. X. 1920. Homogensp. 50. Einsatz II. sp. 29. Einsatz II.

\begin{tabular}{c|c|c|c|c|c|c|c|c|c|c|c}
\hline Lh & W. B. & 643 & 517,5 & Lh & W. B. & 633 & 512 & Ih & W. B. & 623 & 507 \\
\hline 559 & 1240 & 172 & 288 & 559 & 1330 & 106 & 354 & 559 & 1700 & 67 & 393 \\
569 & $\mathbf{1 4 1 0}$ & 207 & $\mathbf{2 5 3}$ & 569 & $\mathbf{1 7 0 0}$ & $\mathbf{1 2 0}$ & $\mathbf{3 4 0}$ & 569 & $\mathbf{1 9 7 0}$ & $\mathbf{7 8}$ & 382 \\
574 & $\mathbf{1 1 5 0}$ & 237 & $\mathbf{2 2 3}$ & $\mathbf{5 7 4}$ & $\mathbf{1 7 3 0}$ & $\mathbf{1 2 7}$ & $\mathbf{3 3 3}$ & 574 & $\mathbf{1 5 3 0}$ & 88 & $\mathbf{3 7 2}$ \\
579 & 980 & 275 & 185 & $\mathbf{5 7 9}$ & $\mathbf{1 3 5 0}$ & $\mathbf{1 4 4}$ & $\mathbf{3 1 6}$ & 579 & 1370 & 99 & $\mathbf{3 6 1}$ \\
589 & $\mathbf{7 8 0}$ & 330 & $\mathbf{1 3 0}$ & 589 & 1220 & $\mathbf{1 9 5}$ & $\mathbf{2 6 5}$ & & & &
\end{tabular}

g') Gleichung 612+501. 7.X. 1920. Homogensp. 10. Einsatz II.

\begin{tabular}{c|r|r|r}
\hline \hline Lh & W. B. & 612 & \multicolumn{1}{|c}{501} \\
\hline 569 & $\mathbf{3 4 \gamma 0}$ & 56 & 404 \\
574 & 3380 & 64 & 396 \\
579 & 865 & 79 & 381 \\
589 & 640 & 107 & 353
\end{tabular}

Reihe III.

$\mathrm{a}^{\prime \prime}$ ) Gleichung $625+542$. 20. II. 1921. Einsatz III.

\begin{tabular}{c|c|c|c}
\hline \hline Lh & W. B. & 625 & 542 \\
\hline 560 & 20 & 152,0 & 298,0 \\
570 & $\mathbf{4 3}$ & 166,0 & 284,0 \\
580 & 36 & 218,5 & 231,5 \\
590 & 10 & 265,5 & 184,5
\end{tabular}

B. Grüngleichungen:

Reihe I.

a) Gleichung $561+487$. b) Gleichung $570+494$. c) Gleichung $574+500$. 20. XI. 1920. Einsatz I. 28. XI. 1920. Einsatz I. 21. V. 1921. Einsatz I.

\begin{tabular}{l|r|r|r|r|r|r|r|r|r|r|r|r}
\hline \hline Lh & W. B. & 561 & 487 & Lh & w. B. & 570 & 494 & Lh & W. B. & 574 & 500 \\
\hline 510 & 300 & 73 & 377 & 510 & 220 & 89 & 361 & 518 & 80 & 94 & 136 \\
520 & 324 & 93 & 357 & 520 & 247 & 113 & 337 & 530 & 130 & 145 & 85 \\
530 & $\mathbf{3 7 6}$ & 118 & 332 & 530 & $\mathbf{3 7 8}$ & 147 & 303 & 540 & 100 & 175 & 55 \\
540 & 308 & 160 & 290 & 540 & 306 & 181 & 269 & 560 & 50 & 189 & 41
\end{tabular}

d) Gleichung $550+480$. 21. XI. 1920 .

Einsatz I.

\begin{tabular}{c|c|c|c}
\hline \hline Lh & W. B. & 650 & 480 \\
\hdashline 510 & 223 & 131 & 319 \\
520 & 228 & 173 & 277 \\
525 & $\mathbf{2 3 0}$ & 184 & 266 \\
530 & 226 & 202 & 248 \\
540 & 170 & 247 & 203
\end{tabular}


Tabelle II (Fortsetzung).

Reihe II.

a') Gleichung $579+485$. b') Gleichung $564+476 . \quad$ c $^{\prime}$ ) Gleichung $550+468$. 13. X. 1920. Einsatz II. 15. X. 1920. Einsatz II. 9. XI. 1920. Einsatz II.

\begin{tabular}{|c|c|c|c|c|c|c|c|c|c|c|c|}
\hline Lhe & W. B. & 579 & 485 & Lh & W. B. & 564 & 476 & $\mathrm{Lh}$ & W. B. & $5 \check{0} 0$ & 468 \\
\hline 519 & 1230 & 78 & 382 & 519 & 550 & 105 & 355 & 490 & 88 & 41 & 429 \\
\hline 529 & 1580 & 84 & 376 & 529 & 800 & 117 & 343 & 500 & 71 & 109 & 361 \\
\hline 539 & 2500 & 88 & 372 & 539 & 700 & 155 & 305 & 510 & 197 & 160 & 310 \\
\hline 549 & 1100 & 93 & 367 & 549 & 550 & 204 & 256 & 520 & 288 & 195 & 275 \\
\hline 559 & 840 & 101 & 359 & & & & & 530 & 353 & 243 & 227 \\
\hline .569 & 800 & 125 & 335 & & & & & 540 & 184 & 316 & 154 \\
\hline
\end{tabular}

\section{Blaugleichungen:}

Reihe I.

a) Gleichung $520+461$. b) Gleichung $510+455$. c) Gleichung $500+449$. 6. XII. 1920. Einsatz I. 10. XI. 1920. Einsatz I. 8. XI. 1920. Einsatz I.

\begin{tabular}{r|r|r|r|r|r|r|r|r|r|r|r}
\hline Lh & W. B. & 520 & \multicolumn{1}{|c|}{461} & Lh & W. B. & 510 & 455 & Lh & W. B. & 500 & 449 \\
\hline 470 & 83 & 70 & 380 & 460 & 3 & 12 & 438 & 460 & 38 & 40 & $\mathbf{4 1 0}$ \\
480 & $\mathbf{8 7}$ & 118 & 332 & 470 & 28 & 87 & 363 & 470 & $\mathbf{4 7}$ & 115 & $\mathbf{3 3 5}$ \\
490 & 77 & 213 & 237 & 480 & 23 & 131 & 319 & 480 & 33 & 161 & $\mathbf{2 8 9}$ \\
500 & 53 & 321 & 129 & 490 & 0 & 151 & 299 & 490 & 26 & 278 & $\mathbf{1 7 4}$ \\
510 & 39 & 426 & 24 & 500 & 0 & 212 & 238 & & & &
\end{tabular}

d) Gleichung $490+443$. 9. XII. 1920. e) Gleichung $480+440$. 16. I. 1921 Einsatz I. Einsatz I.

\begin{tabular}{r|c|c|c|c|c|c|c}
\hline $\mathrm{Lh}$ & W. B. & 490 & 443 & Lh & W. B. & 480 & 440 \\
\hline \hline 50 & 18 & 30 & 420 & 460 & 36 & 122 & 328 \\
160 & 34 & 110 & 340 & 465 & 43 & 168 & 282 \\
471 & 49 & 185 & 265 & 470 & 45 & 218 & 232 \\
480 & 28 & 251 & 199 & 475 & 38 & 316 & 134
\end{tabular}

Reihe II.

a') Gl. $520+451$. b') Gl. $500+440 . \quad$ c$\left.^{\prime}\right)$ Gl. $490+437$. d') Gl. $480+434$. 17. X. 20. Eins. II. 23. X. 20. Eins. II. 24. X. 20. Eins. II. 28. X. 20. Eins. II.

\begin{tabular}{|c|c|c|c|c|c|c|c|c|c|c|c|c|c|c|c|}
\hline $\mathrm{Lh}$ & W. B. & 520 & 451 & $\mathrm{Lh}$ & W. B. & 500 & 440 & $\mathrm{Lh}$ & w. B. & 490 & 437 & $\operatorname{Lh}$ & W. B. & 480 & 434 \\
\hline 160 & 36 & 3 & 467 & 450 & 0 & 6 & 464 & $4 \breve{50}$ & 0 & 11 & 459 & 450 & 10 & 462 & 8 \\
\hline 70 & 90 & 44 & 426 & 460 & 21 & 29 & 441 & 460 & 20 & 38 & 432 & 460 & 13 & 433 & 37 \\
\hline 480 & 66 & 67 & 403 & 470 & 53 & 64 & 406 & 465 & 48 & 72 & 398 & 465 & 30 & 372 & 98 \\
\hline 490 & 58 & 146 & 324 & $\mathbf{4 8 0}$ & 28 & 137 & 333 & 470 & 60 & 101 & 369 & 470 & 26 & 314 & 156 \\
\hline 500 & 26 & 239 & 231 & 490 & 0 & 240 & 230 & 475 & 33 & 150 & 320 & & & & \\
\hline 510 & 0 & 360 & 110 & & & & & 480 & 0 & 219 & 251 & & & & \\
\hline
\end{tabular}

Pflügers Archiv f. d. ges. Physiol. Bd. 194. 
Tabelle II (Fortsetzung).

Reihe III.

a") Gleichung $493+455$. Eirisatz III.

\begin{tabular}{c|c|c|c}
\hline Lh & W. B. & 493 & 455 \\
\hline 465 & 0 & 47,5 & 402,5 \\
470 & 36 & 73,0 & 377.0 \\
475 & $\mathbf{5 0}$ & 83,0 & 367,0 \\
480 & 11 & 89,5 & 360,5
\end{tabular}

D. Gleichungen mït zwei Maxima:

I. Gleichung $589+501$. 10. III. 1921. II. Gleichung $542+456$. 14. III. 1921. Einsatz III.

Einsatz IV.

\begin{tabular}{c|c|r|r|r|r|r|r}
\hline Lh & W. B. & 589 & 501 & th & W. B. & 542 & 456 \\
\hline 520 & 24 & 99 & 301 & 463 & 88 & 3 & 377 \\
530 & $\mathbf{5 4}$ & 160 & 240 & 470 & $\mathbf{1 4 0}$ & 17 & 363 \\
540 & 30 & 280 & 120 & 480 & 118 & 37 & 343 \\
560 & 54 & 302 & 98 & 490 & 177 & 57 & 323 \\
570 & $\mathbf{6 2}$ & 334 & 66 & 510 & 500 & 147 & 233 \\
580 & 23 & 353 & 47 & 520 & $\mathbf{8 7 6}$ & 155 & 225 \\
& & & & 530 & $\mathbf{7 6 8}$ & 190 & 190
\end{tabular}

Es wurden dann Gleichungen gesucht, bei denen keine Weilmischung nötig war, also Binär-Homogen-Gleichungen von vollständigem Geltungsgrade $a \cdot p+b \cdot q=c \cdot r$. An solchen waren für mein rechtes Auge möglich:

Tabelle III.

Rot-Gelbgleichung.

1. Gl. $680+576$.

8. II. 1921.
Blau-Grüngleichung.

2. G1. $525+473$.

3. II. 1921.
Rot-Blaugleichung.

3. Gleichung $470+435$. 20. I. 1921 .

\begin{tabular}{l|r|r|r|r|r|r|r|r|r|r|r}
\hline Lh & W. B. & 680 & 576 & Lh & w. B. & 525 & 473 & Lh & W. B. & 470 & 435 \\
\hline 580 & 0 & 324 & 126 & 485 & 0 & 40 & 410 & 440 & 0 & 2 & 448 \\
590 & 0 & 382 & 68 & 495 & 0 & 84 & 366 & 450 & 0 & 26 & $\mathbf{4 2 4}$ \\
610 & 0 & 406 & 44 & 505 & 0 & 168 & 282 & 460 & 0 & 80 & 370 \\
630 & 0 & 414 & 36 & 515 & 0 & 246 & 204 & & & & \\
650 & 0 & 427 & 23 & & & & & & & &
\end{tabular}

Wenn ich die Ergebnisse meiner gesamten Binär-Homogengleichungen in eine Tabelle (Tab. IV) zusammenfasse, so ergibt sich folgende Übersicht: 
Tabelle IV.

\begin{tabular}{|c|c|c|c|}
\hline $\begin{array}{l}\text { Binärmischungs- } \\
\text { komponenten }\end{array}$ & $\begin{array}{l}\text { Arithmet. Mittel } \\
\text { d. Komponenten }\end{array}$ & $\begin{array}{c}\text { Sättigungsdifferenz- } \\
\text { maximum }{ }^{1} \text { ) }\end{array}$ & Anmerkungen \\
\hline $690+567$ & 628,5 & 576 & Mittelwert 570,8 \\
\hline $678+534$ & 606 & 570 & \\
\hline $670+557$ & 613,5 & 576 & \\
\hline $664+528$ & 596 & 572 & \\
\hline $655+547$ & 601 & 570 & \\
\hline $653+522$ & 587,5 & 570,5 & \\
\hline $643+541$ & 592 & 570 & \\
\hline $643+517$ & 580 & 567,5 & \\
\hline $633+535$ & 584 & 570,5 & \\
\hline $633+512$ & 572,5 & 571,5 & \\
\hline $625+542$ & 583,5 & 575 & \\
\hline $623+527$ & 575 & 569,5 & \\
\hline $623+507$ & 565 & 567 & \\
\hline $612+521$ & 566,5 & 569 & \\
\hline $612+501$ & 556,5 & ca. 564 & sehr ungesättigt \\
\hline $602+516$ & 559 & 569 & 2018 \\
\hline $592+508$ & 550 & 568 & \\
\hline $583+502$ & 542,5 & 564 & \\
\hline $579+485$ & 532 & 537 & Mittelwert $\mathbf{5 3 0}, \mathbf{2}$ \\
\hline $574+500$ & 537 & 532 & \\
\hline $570+494$ & 532 & 528 & \\
\hline $564+476$ & 520 & 530,5 & \\
\hline $561+487$ & 524 & 529,5 & \\
\hline $550+480$ & 515 & 527 & \\
\hline $550+468$ & 509 & 530 & - \\
\hline $540+473$ & 506,5 & $528-530$ & \\
\hline $520+461$ & 490,5 & 476 & Mittelwert $\mathbf{4 7 1 , g}$ \\
\hline $520+451$ & 485,5 & 471,5 & \\
\hline $510+455$ & 482,5 & 473,5 & \\
\hline $500+449$ & 474,5 & 470 & \\
\hline $500+440$ & 470 & 471,5 & \\
\hline $493+455$ & 474 & 474 & ! \\
\hline $490+443$ & 466,5 & 470 & \\
\hline $490+437$ & 463,5 & 469 & \\
\hline $480+440$ & 460 & 468 & \\
\hline $480 \div 434$ & 457 & 467 & \\
\hline $589+501$ & 545 & 528 & \\
\hline $542+456$ & 499 & 470 & \\
\hline
\end{tabular}

1) Aus den vorhergehenden Tabellen lassen sich die Maxima direkt nicht bestimmen. Um Vergleichswerte zu haben, kann man sich einfach der Fiktion bedienen, als sei bei graphischer Darstellung das mittlere Kurvenstück mit dem Maximum etwa eine Parabel und bekommt so eine Reihe von Werten, fiktive Maxima, die natürlich vom tatsächlichen Maximum abweichen, kaum aber einsinnig abweichen. Thr Mittelwert wird ihm also nahekommen. 
Die Tabelle zeigi - ebenso wie die daraus gewonnene graphische Darstellung (Abb. 7) - an 3 Stellen des Spektrums eine unverkennbare Häufung der Sättigungsdifferenzmaxima. Es erhebt sich alsbald die Frage, ob diesen Punkten auch sonstige Eigentümlichkeiten zukommen. Das Naheliegendste schien das Aufsuchen der Orte der Urfarben Herings zum Zwecke des Vergleiches zu sein. Dies konnte eimmal direkt geschehen durch Empfindungsanalyse bei Betrachtung eines homogenen Feldes wie es Westphal ${ }^{1}$ ) getan. Oder indirekt durch Untersuchung, ob sich der Farbenton bei zunehmend indirekter Betrachtung ändert, wie es

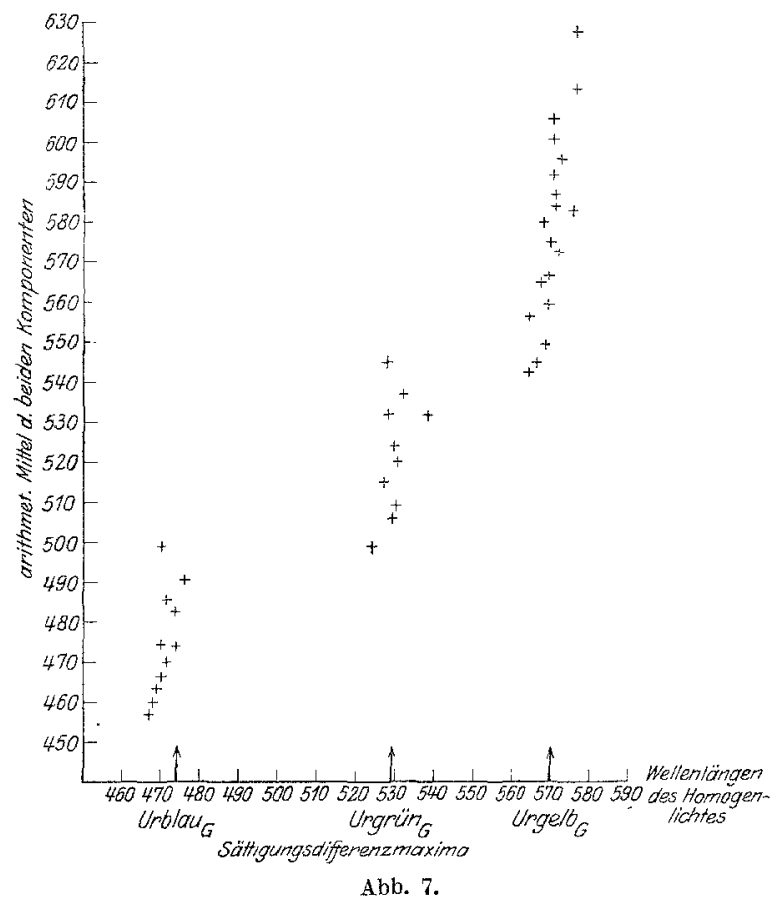

Hess $\left.^{2}\right)$ vornahm. Die Methode von Goldytsch ${ }^{3}$ ) mit Hilfe des Kontrastes farbloser Lichter scheidet deshalb aus, weil sie einander kontrastivfordernde Farben gibt und nicht die Gegenfarben ${ }^{4}$ ). Die Bestimmungen lieferten als Mittel aus den Ergebnissen an verschiedenen Tagen.

$$
\begin{array}{lll}
\text { Urgelb . . . . } & \mathbf{5 7 0} \pm \mathbf{2} \\
\text { Urgrün. . . . } & \mathbf{5 2 9} 3 \\
\text { Urblau. . . . } & 474
\end{array}
$$

1) H. Westphal, Unmittelbare Bestimmung der Uxfarben. Zeitschr. f. Sinnesphysiol. 44, 182. 1909.

9) C. Hess, Graefes Arch. f. Ophthalmol. 35, H. 4, S. 1. 1889.

3) L. Goldytsch, Zeitschr. f. Biol. 6r, 35. 1917.

1) A. Tschermak, Utber das Verhältnis von Gegenfarbe, Kompensationsfarbe und Kontrastfarbe. Pflügers Arch. f. d. ges. Physiol, 11\%, 473. 1907. 
Diese Werte stammen aus der Zeit der oben angeführten Gleichungsreihen. Spätere Reihen seien hier detailliert angeführt.

Tabelle $V$.

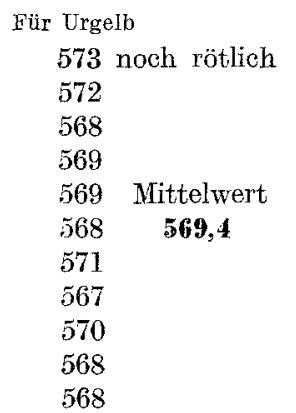

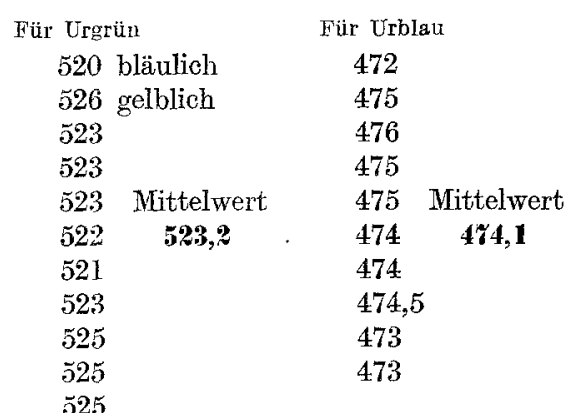

Die indirekte Bestimmung ergab ca. 567, 522, 466. Diese drei Reihen seien hier angeführt, weil das Urgrün eine Besonderheit zeigt. Sie wurden 3 Monate nach den obigen Maximabestimmungen durchgeführt - gleichzeitig mit der letzten Gleichung $\left(D_{I I}\right.$ in Tab. II) -- und zeigten eine starke Abweichung gegenüber den älteren Bestimmungen. Damals hatte sich als Mittelwert der Urgrünbestimmungen $529 \mu \mu$ ergeben, diesmal aber 523 und besonders auffällig erschien es, daß sich in Übereinstimmung damit aus obiger Gleichung ( $D_{I I}$ Tab. II) ebenfalls ein viel tiefer liegendes Maximum ergibt $(524 \mu \mu)$. Daraufhin wurden neuerlich Gleichungsreihen durchgeführt, um diesen Befund zu untersuchen. Bei einer Reihe habe ich, um jedes suggestive Moment auszuschalten, bei verschobener Poggendorf-Ablesung gearbeitet und nachher erst geeicht und die zugehörige Wellenlänge bestimmt. Die nächste Tabelle zeigt die Resultate dreier peinlichst durchgeführter Gleichungsreihen.

Tabelle VI.

1. G1. $550+490$. 26. VI. 1921.
2. G1. $540+483$.

29. VI. 1921.
3. G1. $561+498$.

9. VI. 1921 .

\begin{tabular}{c|r|r|r|r|r|r|r|r|r|r|r}
\hline \hline Lh & W. B. & 490 & 550 & Lh & W. B. & 483 & 540 & Lh & W. B. & 561 & 498 \\
\hline 520 & 450 & 141 & 94 & 510 & 180 & 130 & 105 & 512 & 160 & 18 & 217 \\
525 & $\mathbf{5 0 0}$ & 116 & 119 & 521 & $\mathbf{3 5 0}$ & 105 & 130 & 523 & $\mathbf{2 4 4}$ & 35 & 200 \\
531 & 390 & 90 & 145 & 530 & 180 & 68 & 167 & 530 & 120 & 53 & $\mathbf{1 8 2}$
\end{tabular}

Danach besteht kein $Z$ weifel, daß das Sättigungsdifferenzmaximum im Grün zur Zeit der zweiten, detaillierten Bestimmung des Urgrüns mit 523,2 als heterotopem Mittelwert ebenfalls verlagert war und als mit eben diesem Punkte übereinstimmend bezeichnet werden darf.

Wodurch diese interessante, völlig parallele zeitliche Änderung meines Sehorgans bedingt ist, vermag ich nicht anzugeben. Einfach 
auf adaptative Veränderungen läßt sie sich wohl nicht zurückführen; daß sie mit der Anomalie meines Farbensinnes im Sinne einer extremen Blausichtigkeit zusammenhängen kann, will ich nicht ausschließen, doch zeigen auch sog. Normale ein ähnliches Wandern des Urgrüns, wie sie bereits $A$. Tschermak an sich bemerkt hat und die nachfolgenden Resultate näher illustrieren mögen. Jedenfalls gedenke ich diese scheinbar spontane Variabilität einer gesonderten Untersuchung zu unterwerfen. Sie wurde auch bei einem relativ Gelbsichtigen festgestellt. Herr Dr. M. H. Fischer, dessen Liebenswürdigkeit ich die folgenden Gleichungen verdanke, ist relativ stark gelbsichtig, zeigt völlig normalen Farbensinn, liest alle Tafeln Stillings ohne Mühe, stellt am Anomaloskop die sog. normale Gleichung ein (56 bei unserem Instrument). Auch sei in dieser Beziehung auf die eingangs gegebene Vergleichstabelle hingewiesen. - Ich führe hier seine folgenden Beobachtungen an.

A. Gelbgleichungen von Dr. M. H. F.

1. Gl. $625+542$.

2. Gl. $670+535$.

\begin{tabular}{c|c|c|c|c|c|c|c}
\hline \hline Lh & W. B. & 625 & 542 & Lh & W. B. & 535 & 6 (670 \\
\hline \hline 550 & 0 & 68 & 382 & 580 & 0 & 83 & 317 \\
570 & 0 & 89 & 361 & 590 & $\mathbf{8 7}$ & 50 & 350 \\
580 & 0 & 120,5 & 329,5 & 600 & 34 & 36 & 364 \\
590 & 0 & 167 & 283 & & & &
\end{tabular}

Die Urgelbbestimmung ergab mit Gleichung 2 übereinstimmend 590. Interessant ist, daß in Gleichung 1 keine Weißbeimischung erforderlich war, ebenso wie es $E$. Hering für den Gelbsichtigen B. angibt $\left.{ }^{1}\right)$. Bemerkt sei aber, daß bei der Einstellung die Gleichung $L 625+L 542=L 590$ die längste Zeit beanspruchte und lange nicht befriedigte, was wohl dahin zu deuten ist, daß eine kaum merkliche Sättigungsdifferenz vor$\left.\operatorname{lag}^{2}\right)$. Inwieweit die Empfindlichkeit für Gelbgleichungen, was Sättigung anbelangt, einen Typencharakter im Sinne A. Tschermaks ${ }^{3}$ ) darstellt, muß erst eine statistische Untersuchung lehren.

B. Blaugleichungen von Dr. M. H.F.

Gl. $503+441$. Urblaubestimmung: 476 .

\begin{tabular}{l|r|r|r}
\hline \hline Lh & W. B. & \multicolumn{1}{c}{503} & \multicolumn{1}{c}{4.11} \\
\hline \hline 460 & 10 & 42 & 358 \\
470 & 30 & 74 & 326 \\
480 & $\mathbf{3 8}$ & 99 & 301 \\
490 & 32 & 113 & 287
\end{tabular}

1) E. Hering a. a. O.; A. Tschermak a. a. 0.

9) H. Aubert a. a. O.; Hering, Ủber den Einfluß der Macula lutea auf spektrale Farbengleichungen. Pflügers Arch. f. d. ges. Physiol. 54, 277. 1893.

3) A. Tschermak a. a. O., Ergebn. d. Physiol. 1, 743. 1902. 
Die Grüngleichungen sind nun wieder sehr interessant:

1. Gl. $560+495$.

\begin{tabular}{c|c|c|c}
\hline Lh & W. B. & \multicolumn{1}{|c|}{560} & \multicolumn{1}{|c}{495} \\
\hline 520 & 203 & $\mathbf{1 1 5 , 5}$ & $\mathbf{3 3 4 , 5}$ \\
$\mathbf{5 3 0}$ & $\mathbf{3 0 3}$ & $\mathbf{1 4 4}$ & $\mathbf{3 0 6}$ \\
$\mathbf{5 4 0}$ & 223 & $\mathbf{1 5 9}$ & $\mathbf{2 9 1}$
\end{tabular}

Die später, doch am selben Vormittage direkt angestellte Urfarbenbestimmung ergab 520, also einen weitab liegenden Wert:

2. Gl. $560+495$.

\begin{tabular}{c|c|c|c}
\hline \hline Lh & w. B. & 560 & \multicolumn{1}{|c|}{495} \\
\hline \hline 516 & 160 & 121,5 & 328,5 \\
526 & $\mathbf{2 3 8}$ & 137 & 313 \\
535 & 202 & 148 & 302
\end{tabular}

- sofort nachher wird das Urgrün bestimmt und ergibt diesmal 529. Daraufhin wurde vor und nach Einstellung einer jeden Gleichung die Urfarbenbestimmung durchgeführt:

3. G1. $560+495$.

\begin{tabular}{c|c|c|c|c}
\hline \hline Lh & & W. B. & 560 & 495 \\
\hline \hline \multirow{2}{*}{520} & Urgr. 520 & & & \\
\multirow{2}{*}{525} & Urgr. 525 & 200 & 122 & 328 \\
530 & Urgr. 525 & $\mathbf{2 1 0}$ & 126 & 324 \\
& Urgr. 525 & 171 & 128 & 324
\end{tabular}

Mittel 525 .

Auch die Ursache des obenerwähnten Lagewechsels des Urgrüns, der bei Dr. M.H.F. relativ plötzlich eintrat und zufällig erhascht wurde, muß vorläufig als ungeklärt bezeichnet werden. Jedenfalls variieren Urfarbigkeit und maximale Sättigungsdifferenz völlig parallel, ja sie betreffen jeweilig dasselbe Spektrallicht.

Es sei, um alles Zahlenmäßige gleich hier zusammenzufassen, noch folgendes betont.

1. Außer der oben gegebenen Tabelle zur Charakterisierung meines Farbensinnes ist hervorzuheben, daß die Strecke an den beiden Spektrumsenden, in denen zwischen Homogenlichtern vollständige Gleichungen durch einfache Intensitätsänderungen zu erhalten sind (die Königschen „Endstrecken"), für mich einerseits nicht unter $670 \mu \mu$ herab, andererseits nicht über 428 hinaufreichen. 
2. Zur liethode sei noch angeführt, daß bei Änderung der Kollimatorspaltweite innerhalb der verwendeten Weiten der Farbenton sich nicht merklich änderte. Dies ließ sich am besten daran erkennen, daß die Grenze der Endstrecke erhalten blieb, wenn die Spaltweite des einen Kollimators variiert wurde. Ich führe diese Charakteristik an, weil Erweiterung des Kollimatorspaltes etwa um 570 Änderungen auch in der Sättigung der Farbe bewirkt. Hier scheint überhaupt im ersten Moment eine schwerwiegende Fehlerquelle der Methodik zu liegen, da ja schon abhängig von der Kollimatorspaltweite die Sättigung sich ändern wird. Bei gekoppelten Spalten muß die Reinheit einer Komponente immer auf Kosten der anderen gehen, doch wird die durch die Spaltweite bedingte Sättigungsdifferenz um so stärker hervortreten, je weiter einer der gekoppelten Spalte ist, d. h. gegen beide Enden einer Reihe. Die Sättigung wird gegen beide Enden abnehmen, ohne ein Maximum zu zeigen. Und damit ist schon die Brauchbarkeit einer Reihe bestimmt. Eine Reihe wie die folgende ist unbrauchbar:

$$
\text { G1. } 535+463 \text {. }
$$

$\begin{array}{cc}\text { Wellenlănge } & \text { Weißbeimischung } \\ 525 & \mathbf{1 8 0} \\ 510 & 90 \\ 495 & 64 \\ 480 & \mathbf{2 3 0}\end{array}$

Jedenfalls geht aus all den angetührten Reihen die enge Verknüpfung von Sättigungsdifferenzmaximum und Urfarbe hervor.

IV. Folgerungen für die Theorien des Farbensinnes.

Aus den angeführten Versuchen ergibt sich, daß die Sättigungsdifferenzmaxima an ganz bestimmten Stellen im Spektrum auftreten, die je nach Individuum Variationen aufweisen. Es sind 3 solche Punkte gefunden worden (vgl. Abb. 7). Wenn man unter denselben Umständen und um dieselbe Zeit die Heringschen Urfarben (Urgelb, Urgrün, Urblau) im Spektrum aufsucht, so findet man diese 3 Kardinalpunkte genau an jenen Stellen. Die Orte der Urfarben sowohl als anch der Maxima fallen nicht etwa zufällig zusammen, da 1 . bei zeitlicher Änderung der Stelle der Urfarbenorte beim selben Individuum die Maxima dieselben Änderungen erfahren; 2. diese Koppelung auch bei verschiedenen Individuen bzw. Typenvertretern besteht.

Wendet man auf diese Ergebnisse die eingangs gemachten theoretischen Auseinandersetzungen an und interpretiert sie danach, so sagen sie aus, daß die durch die Spektralfarben dargestellte Begrenzungslinie der Farbenfläche an diesen Punkten plötzliche Richtungsänderungen zeigt, schematisch gesprochen, Eckpunkte erkennen läßt. Schematisch deshalb, weil nach der mathematischen Analyse unsere Untersuchung nicht ein 
Kriterium für Eckpunkte, sondern nur für Krümmungsmaxima liefert. Daß die Krümmungsänderung einer Ecke mindestens sehr angenähert entspricht, geht daraus hervor, daß Binärgemische aus solchen Lichtern, welche zwei in der Spektralfarbenreihe benachbarten Urfarben entsprechen, verglichen mit Homogenlichtern keine Sättigungsdifferenzen erkennen lassen (vgl. Tab. III), also die Farben der Gemische und die Spektralfarben auf der gleichen Linie bzw. einer (relativen) Geraden der Farbenfläche liegen ${ }^{1}$ ). Infolgedessen ist man imstande, aus den Spektrallichtern des Urgelb, Urgrün und Urblau sämtliche dazwischenliegenden Farbentöne und Sättigungsstufen von Weiß bis zur Sättigung der Farben spektraler Homogenlichter durch physikalische Mischung zu erhalten. Es fehlen dabei aber noch alle roten Töne. Von den gelbroten und violetten Tönen, die das Spektrum liefert, zeigen obige Versuche (siehe Gleichungen ohne Sättigungsdifferenz), daß sie längs kürzester Linien angeordnet sind. Entwirft man nach alledem eine schematische Figur für die Spektralfarbenreihe, so kann sie nur folgende Form haben (Abb.8).

Wenn man also die Mindestzahl spektraler Lichter sucht, aus denen sämtliche durch Mischung aller möglichen Spektrallichter darstellbaren Farbentöne und Sättigungsstufen erhalten werden können, so ergeben sich fünf, nämlich rotes Ende, Urgelblicht, Urgrünlicht, Urblaulicht, violettes Ende.

Man kann die Frage aber auch so stellen: Wieviel Receptoren oder Elementarreagenten im Substrate muß man mindestens annehmen, um den Resultaten ohne

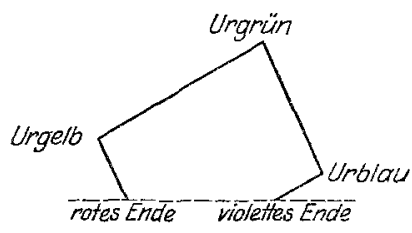

Abb. 8 . weitere Hilfshypothese gerecht zu werden. Diese Fragestellung ist offenbar unabhängig von dem Spektralcharakter der Lichter; es könnte ja Receptoren geben, die allein niemals von einem Spektrallicht, sondern nur von Mischungen gereizt werden. Zieht man dies mit in Betracht, so braucht man in voriger Figur nur die Geraden, welche die Gelbrot- und Violettstrecke symbolisieren, bis zu ihrem Schnittpunkte zu verlängern. Die Lage dieses Punktes kann nicht zweifelhaft sein: er entspricht Herings Urrot. Denn nur dann ist der Schwerpunkt der Farbenfläche auch der Ort des Weiß. Die spektrale Farbenreihe erfüllt allerdings nur einen Teil des Konturs der viereckigen Farbenfläche: die Urrotecke sowie eine kurze Strecke schwachgelblicher Töne und die längere Strecke der Purpurtöne fehlen im Spektrum, wie dies bereits Newton, Goethe und E. Hering betont haben.

1) Für die Spelztralregion Gelb bis Grün war es mir aus technischen Gründen (Breite der Einsatzstücke) nicht möglich, eine Gleichungsreihe durchzuführen, doch geht aus der gesamten Literatur hervor, daß auch hier tadellose Mischungen möglich sind. 
Das Farbenviereck (Abb. 9) erscheint in bezug auf das Spektrum nicht geschlossen, vielmehr - aus unbekannten Gründen - durch Fehlen der vierten Ecke zu einem Linienzuge mit bloß 3 Ecken, jedoch mit unverkennbarer Abbiegung und Konvergenz des Linienzuges jenseits der Urgelb- wie jenseits der Urblauecke reduziert. Ob die in der vollständigen Goethe-Heringschen Farbenschaar ausgezeichnete Urrotecke nicht doch auch für die spektrale Lichterreihe unter gewissen Umständen in sinnfällige Erscheinung treten könnte, bleibe hier dahingestelltt ${ }^{1}$ ). Die beiden Maxima im Farbenviereck, denen Urgelb und Urblau zugeordnet erscheinen, sind komplementär, also müssen es auch die beiden anderen

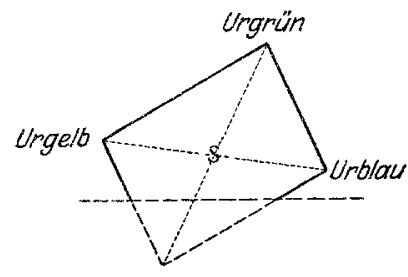

Abb. 9. sein. Es sind also nicht 4 einzelne, voneinander unabhängige, sondern 4 paarweise gekoppelte Receptoren oder Elementarreagenten zu erschließen.

Es scheint hier der Ort zu sein, kurz noch einmal die beiden großen Theorien des Farbensinnes zu streifen. Grundlage jeder Farbentheorie muß die Tatsache der dreifachen Ausdehnung des Farbensystems sein, die aus der Richtigkeit von Newtons Regel folgt. Unbestimmt erscheinen dabei noch die 3 Koordinaten; sie sind der Theorie überlassen. Daraus geht hervor, daß die einfache Darstellbarkeit mit 3 Koordinaten keinerlei Beweisgrund für irgendeine Theorie abgeben kann. Nichtsdestoweniger haben viele Physiker, seitdem Maxwell ${ }^{2}$ ) den Beweis für Newtons Regel erbrachte und diesen sofort als Beweis der Youngschen Theorie ansprach, einer solchen unzulässigen Argumentation beigepflichtet. So schloß v. Bezold ${ }^{3}$ ), welcher Formeln für die Komplementärfarben ableitete, daraus, daß die einfachsten Formeln sich aus der Young-Helmholtzschen Theorie ergeben, auf eben deren Richtigkeit. Was er aber zur Voraussetzung macht, erweist sich bei näherem Zusehen nicht als Young-Helmholtzsche Theorie, sondern einfach als jene Grundtatsache der dreidimensionalen Ausdehnung des Farbensystems. Auch die neuern Daten, die König und Dieterici ${ }^{4}$ ), endlich $F$. Exner ${ }^{5}$ )

1) Der Frage des Einflusses der Dunkeladaptation auf die Farbentonverteilung im Spektrum soll eine spätere Institutsarbeit gelten.

$\left.{ }^{3}\right)$ I. C. Maxwell a. a. O. und Experiments on Colour as perceived by the Eye. Edinb. Transact. 21 (II), 275ff. 1855.

3) W. v. Bezold, Über das Gesetz der Farbenmischung und die physiologischen Grundfarben. Poggendorfs Annalen 150, 71. 1873.

$\left.{ }^{4}\right)$ A. König und C. Dieterici. Die Grundempfindungen und ihre Intensitätsverteilung im Spektrum. Abt. d. Preuß. Akad. d. Wiss. 1886, S. 805; Die Grundempfindungen in normalen und anormalen Farbensystemen und ihre Intensitätsverteilung im Spektrum. Zeitschr. f. Psychol. u. Physiol. d. Sinnesorg. 4, 241. 1892.

5) F. Exner, Grundempfindungen im Helmholtzschen Farbensystem. Sitzungsber. d. Wien. Akad. d. Wiss., Math.-naturw. Kl. Abt. I. 129, 27. 1920. 
liefern, sind nur Ausdruck der Räumlichkeit des Systems bei willkürlicher Wahl der Koordinaten. Jeder dieser "Beweise" rührt aber an keiner Theorie, da jederzeit Transformationsgleichungen gefunden werden können, welche die in dem einen System dargestellten Beziehungen in dem anderen definieren. Helmholtz selbst hat eine Art solcher Gleichungen zur Transformation seines Systems in ein System von der Art des Heringschen in seiner physiologischen Optik gegeben. Ein Grund, die zahlenmäßigen Mischungsergebnisse zum „Beweise“ für die YoungHelmholtzsche Theorie heranzuziehen, mochte für manchen Vertreter dieser Auffassungsweise auch darin gegeben erscheinen, daß E. Hering selbst eine bezügliche Verwertung oder Auseinandersetzung unterließ, und daß im Vermeiden einer Berücksichtigung dieser für den Physiker klarsten Beziehungen des Messens eine Schwäche seines Systems erblickt werden konnte. Der Physiker kann eben ein reduziertes konstantes Sehorgan fingieren, welches sämtliche eindringenden Wellenzüge in 3 Komponenten zerlegt. Damit ist für ihn alles bereinigt. Das Sehorgan wird hierbei behandelt wie ein konstanter physikalischer Apparat, die Intensitäten der Erregung werden als glatt darstellbar erachtet durch die Intensitäten des Reizes. Alles andere wird zum „Versuchsfehler".

Da für Quantitätsbeziehungen nur die Dimension maßgebend ist, Quantitätsbeziehungen aber, solange eben das Lebendige nicht mathematisch faßbar ist, nur als Fiktionen existieren, genügen dem Physiker die Young-Helmholtzschen Annahmen. Dem Physiker erscheinen dieselben nicht nur völlig ausreichend, sondern sogar vorteilhaft: er macht die Fiktion der Konstanz des Sehorganes und braucht als Variable des dreidimensionalen Systems - denn für Quantitätsbeziehungen ist ja die Form unwesentlich - Variable, die möglichst den Variablen des Reizes entsprechen: Wellenlänge $=$ Farbenton, Intensität $=$ Helligkeit und, was noch übrigbleibt, technisch ausgedrückt durch die Beimischung von „,weißem" Lichte = Sättigung. Das Heringsche System mag in dieser Beziehung geradezu unbequem erscheinen. Daß der Physiologe all diese Fiktionen durchbrechen $m u \beta$, ist dem Physiker oft nicht klar, ja er ist sogar stolz auf sein Gebäude ${ }^{1}$ ). Es ist also nicht eine Schwäche Herings und seiner Schule, daß sie vermeiden, Quantitätsbeziehungen aufzustellen, sondern nur kritische Beurteilung von Fiktionen.

Gewiß gewinnt der Physiker mit seinen Fiktionen, speziell der ,Konstanz des Sehorgans" eine Basis für die Messung von Reizgrößen. Dem Physiologen kommt es auf das Geschehen im Sehorgan an; er nähme sich sein eigentliches Forschungsgebiet, wollte er von vornherein das

1) F. Exner. Zur Kenntnis des Purkinjeschen Phänomens. Sitzungsber. d. Wien. Akad. d. Wiss. Math. naturw. Kl. Abt. I, 128. 71, 1919; Zur Frage nach der spezifischen Helligkeit der Farben. Zeitschr. f. Sinnesphys. 51, 157. 1921. 
Auge zum physikalischen Apparat reduzieren. Die Reizmessung zeigt ihm nur biologische Variationen, sie ist ihm nur eine zahlenmäßige Charakteristik der an sich nicht meßbaren Erregungsgröße. Für ihn ist auch der "Versuchsfehler" interessant, weil er biologisch ist. Die genauen Zahlen der Reizmessung nützen ihm aber nicht viel, weil er sie nur in ihrer Beziehung zur Erregungsgröße brauchen könnte, für diese aber ein Maßsystem unbekannt ist und ferner die Reizwerte je nach Individuum und Zeit schwanken, was an sich sehr wichtig ist. Denn wenn überhaupt solche Kurven gezeichnet werden sollen, so müBten erst die individuellen Schwankungen nach Mittelwert und mittlerem Fehler charakterisiert sein. All die anderen noch so genau ermittelten Kurven tragen viel zu individuelles Gepräge; wertvoll ist nur ihre allgemeine Form, die Einzelzahlen gebören in eine Personalbeschreibung des Individuums. Aus diesen Erörterungen ergibt sich, kurz gesagt, daß Untersuchungen über Lichtermischung, welche bloß die 3 Dimensionen des Farbensystemes zur Grundlage heranziehen, weder für Young-Helmholtz noch für Hering etwas aussagen. Mischungsuntersuchungen, welche wirklichen Aufschluß dazu bringen können, sind solche, welche, wie schon einleitend hervorgehoben wurde, die Frage betreffen, inwieweit eine endliche Anzahl von Farbenkomponenten genügt, um sämtliche durch äußere Reize auslösbaren Farbenempfindungen zu erzeugen, welche also bestrebt sind, die Form der Umgrenzungslinie der Farbenfläche festzustellen. Ist diese Linie ein Kreis, so sind also unendlich viele Komponenten xötig, alle aber dreifach abhängig, da das ganze System dreidimensional ist. Man könnte sich das dann so vorstellen, daß peripher die Dimensionen auf drei beschränkt werden, zentral, unter Einengung der Größe und Form der Farbenfläche die Erregung auf einzelne aus der großen Zahl der Komponenten beschränkt wird. Wie diese Untersuchung aber beweist, ist die Farbenfläche als ein Viereck zu betrachten. Es genügt also ohne jede Hilfshypothese die Heringsche Gegenfarbentheorie zur Erklärung, da ihre 3 Vektoren: Weiß-Schwarz, Rot-Grün, Gelb-Blau der Dimension, die Koppelung Rot-Grün, Gelb-Blau der Form der Farbenfläche ohne Zwang genügen. Der ersten Forderung genügt auch die Young-Helmhollzsche Theorie, der zweiten jedoch nicht.

Auch sei hier darauf hingewiesen, daß bei Bestimmungen physiologisch charakteristischer Punkte im Spektrum regelmäßig die Heringschen Urpunkte gefunden wurden. Sie entsprechen den Asymptoten und den Scheitelpunkten der Hyperbel der Komplementärfarben nach Helmholtz, sie treten auch bei der vergleichenden Prüfung des Farbentons im direkten und im indirekten Sehen hervor ${ }^{1}$ ). Das Bezold-Brückesche

1) C. Hess, Über Farbensinn im indirekten Sehen. Graefes Arch. f. Ophthalmol. $35(4), 1-62.1889$. 
Phänomen ist, wie schon oben betont, sehr leicht nach der Gegenfarbentheorie zu deuten. F. Exner ${ }^{1}$ ) kommt zu dem theoretischen Schlusse, daß nach der Helmholtzschen Theorie 4 Punkte im Spektrum ihren Farbenton behalten müßten; nach seinen Angaben aber gelingt dies für den bei etwa $\lambda=494 \mu \mu$ postulierten Punkt $c$ nicht, da die Änderung ,,nicht so auffallend, und schwierig zu beobachten sei“. Die Heringsche Theorie fordert einen solchen vierten Punkt nicht, wohl aber einen solchen im Urrot, d. h. außerhalb des Spektrums, im vervollständigten Farbenkreis oder Farbenpolygon. Andererseits findet F. Exner ${ }^{2}$ ), daß als schöne Farben nicht nur Rot, Grün und Blau bezeichnet werden, sondern daß auch reines Gelb „eine besondere Stellung einnimmt", d. h. sich bei den Untersuchungen genau so verhält, wie Rot, Grün und Blau. All das ist um so leichter erklärlich, als die Grundfarben "Grün" und „Blau" für beide Theorien fast identisch sind und das „Rot" von Helmholtz auch nicht viel gegen Gelb von dem Herings abweicht. Die Hauptunterschiede der beiden Theorien liegen aber gar nicht so sehr auf dem Gebiete, das der Physiker zu behandeln pflegt, wenn er farbentheoretisch arbeitet, nämlich im Auswerten der Resultate der Mischungsregel, sondern vor allem im Bewerten der Empfindung als Korrelat physiologischer Vorgänge, wie dies in letzter Zeit speziell $A$. Tschermak ${ }^{3}$ ) klar ausgesprochen hat. Hier soll diese Arbeit ein wenig zur Klärung beitragen. Sie mag vor allem folgendes lehren: Spricht man der Newtonschen Mischungsregel nichts zu, als da $B$ sie Ausdruck lagegeometrischer Symbolik ist, nimmt man sie also in einer Form, die alle anerkennen, und untersucht die so gewonnenen allgemeinen Charakteristika in ihren Beziehungen zu den Resultaten der Empfindungsanalyse, so geben beide, Mischungsversuch und einfache Analyse, übereinstimmende Resultate. Beide sind für den Fall gleichwertig, beide betreffen den Ausdruck physiologischer Vorgänge im Sehorgan als Ganzes, sozusagen des reduzierten Sehorgans, wobei wir die unübersehbare unbekannte Zahl der Differentialvorgänge durch die Fiktion einer einfachen, übersichtlichen, unseren Kenntnissen vor der Hand genügenden Kausalitätsreihe ersetzt haben.

Zum Schlusse möchte ich mir erlauben, Herrn Professor A. Tschermak für die Anregung zu dieser Arbeit und seinen liebenswürdigen Beistand zu jeder Zeit meinen herzlichsten Dank zum Ausdruck zu bringen.

1) F. Exner, Über die Grundempfindungen im Young-Helmholtzschen Farbensystem. Sitzungsber. d. Wien. Akad. d. Wiss, Math.-naturw. K1. Abt. 2 a. 111. 857. 1901.

2) F. Exner, Zur Charakteristik der schönen und häBlichen Farben. Sitzungsber. d. Wien. Akad. d. Wiss., Math.-naturw. Kl. Abt. 2a. 111. 901. 1902.

3) A. Tschermak, Der exakte Subjektivismus. Pflügers Arch. f. d. ges. Physiol. 88, I ff. 1921; auch sep. Berlin 1921. 
526 H. Goldmann: Über den Geltungsgrad spektraler Farbengleichungen.

$$
\text { V. Schlußsätze. }
$$

1. Optische Gleichungen, welche hergestellt wurden am Prager Spektrallichtermischungsapparat nach $E$. Hering zwischen einem homogenen Spektrallicht und einem Gemisch zweier Spektrallichter, die ein urfarbiges Licht (Urgelb, Urgrün, Urblau im Sinne Herings) zwischen sich fassen, zeigen - wenigstens für einen relativ blausichtigen, sog. protanomalen Beobachter - einen unvollständigen Geltungsgrad. Sie erweisen sich als bloße Tongleichungen, in denen die Binärhälfte minder satt bleibt. Nur durch Zumischung weißen Lichtes zur Homogenhälfte ist vollständige Gleichheit zu erzielen.

2. Bei einer ausgedehnten Untersuchung und genau messender Charakterisierung solcher Gleichungen haben sich im Spektrum mit voller Deutlichkeit 3 Häufungsmaxima für Sättigungsdifferenz und damit drei durch optimale Farbsättigung ausgezeichnete Punkte ergeben, welche bei Untersuchung unter gleichen Umständen und zur selben Zeit für das einzelne Individuum mit dessen 3 urfarbigen Spektrallichtern übereinstimmen.

3. Die Lage des Sättigungsmaximums und parallel damit der urfarbigen Stelle im Grün zeigt eine charakteristische Variation.

4. Der Linienzug der Spektralfarben im Farbenfelde bzw. die Umgrenzungslinie ihrer Farbenfläche zeigt demnach entsprechend den 3 urfarbigen Kardinalpunkten 3 deutliche Ecken, zwischen denen (relativ) gerade Strecken verlaufen. Als Farbenfläche ergibt sich nicht ein Dreieck, sondern ein Viereck, dessen vierte Ecke dem im Spektrum fehlenden Urrot entspricht. Es sind 4 paarweise gekoppelte Receptoren oder Elementarreagenten im Sehorgan zu erschließen.

5. Aus der bloßen Tatsache des dreidimensionalen Charakters unseres. Farbensystems und der Gültigkeit des Newtonschen Mischungsgesetzes (d. h. der Mischbarkeit aller Farbentöne aus 3 passend gewählten Lichtern) ist weder für die Heringsche noch für die Young-Helmholtzsche Theorie des Farbensinns etwas Entscheidendes auszusagen. Den Forderungen des Dimensionalcharakters des Systems genügen eben beide. Hingegen entspricht nur die erstere Theorie mit der Statuierung einer Koppelung von je 2 der 4 Grundfarben der durch die vorstehende Untersuchung begründeten Forderung einer Viereckform der Spektralfarbenfläche. 